\title{
ERS statement on standardisation of cardiopulmonary exercise testing in chronic lung diseases
}

\author{
Thomas Radtke (10) ${ }^{1,2}$, Sarah Crook ${ }^{1}$, Georgios Kaltsakas ${ }^{3,4}$, Zafeiris Louvaris ${ }^{5}$, \\ Danilo Berton $^{6}$, Don S. Urquhart (107), Asterios Kampouras (10) ${ }^{8}$, Roberto \\ A. Rabinovich ${ }^{9,10}$, Samuel Verges ${ }^{11}$, Dimitris Kontopidis ${ }^{12}$, Jeanette Boyd ${ }^{13}$, \\ Thomy Tonia ${ }^{14}$, Daniel Langer $\mathbb{0}^{5,15}$, Jana De Brandt ${ }^{16}$, Yvonne M.J. Goërtz ${ }^{17}$, \\ Chris Burtin ${ }^{16}$, Martijn A. Spruit (16 16,17, ${ }^{18}$, Dionne C.W. Braeken ${ }^{17}$, \\ Sauwaluk Dacha ${ }^{5,15,19}$, Frits M.E. Franssen (1) ${ }^{17,18}$, Pierantonio Laveneziana ${ }^{20,21}$, \\ Ernst Eber ${ }^{22}$, Thierry Troosters ${ }^{23,24}$, J. Alberto Neder (1025, Milo A. Puhan (1) ${ }^{1}$, \\ Richard Casaburi ${ }^{26}$, Ioannis Vogiatzis ${ }^{4,27,29}$ and Helge Hebestreit ${ }^{28,29}$
}

@ERSpublications

The document facilitates standardisation of conducting, reporting and interpreting cardiopulmonary exercise tests in chronic lung diseases for comparison of reference data, multi-centre studies and assessment of interventional efficacy. http://bit.ly/31SXeB5

Cite this article as: Radtke T, Crook S, Kaltsakas G, et al. ERS statement on standardisation of cardiopulmonary exercise testing in chronic lung diseases. Eur Respir Rev 2019; 28: 180101 [https://doi.org/ 10.1183/16000617.0101-2018].

ABSTRACT The objective of this document was to standardise published cardiopulmonary exercise testing (CPET) protocols for improved interpretation in clinical settings and multicentre research projects. This document: 1) summarises the protocols and procedures used in published studies focusing on incremental CPET in chronic lung conditions; 2) presents standard incremental protocols for CPET on a stationary cycle ergometer and a treadmill; and 3) provides patients' perspectives on CPET obtained through an online survey supported by the European Lung Foundation. We systematically reviewed published studies obtained from EMBASE, Medline, Scopus, Web of Science and the Cochrane Library from inception to January 2017. Of 7914 identified studies, 595 studies with 26523 subjects were included. The literature supports a test protocol with a resting phase lasting at least $3 \mathrm{~min}$, a 3 -min unloaded phase, and an 8- to 12-min incremental phase with work rate increased linearly at least every minute, followed by a recovery phase of at least 2-3 min. Patients responding to the survey $(n=295)$ perceived CPET as highly beneficial for their diagnostic assessment and informed the Task Force consensus. Future research should focus on the individualised estimation of optimal work rate increments across different lung diseases, and the collection of robust normative data.

Editorial comment in: Eur Respir J 2019; 54: 1901441 [https://doi.org/10.1183/13993003.01441-2019].

This article has supplementary material available from err.ersjournals.com

This article was endorsed by the ERS Executive Committee on 14 August 2019

Provenance: Submitted article, peer reviewed.

Received: 07 May 2019 | Accepted: 16 June 2019

Copyright (CERS 2019. This article is open access and distributed under the terms of the Creative Commons Attribution Non-Commercial Licence 4.0. 


\section{Introduction}

Cardiopulmonary exercise testing (CPET) is useful in the assessment of subjects with chronic lung conditions as it may help to: 1) recognise physiological factors limiting exercise (with or without the presence of psychogenic limiting factors); 2) identify these factors as potential therapeutic targets; 3 ) allow quantification of the level of impairment; 4) assess the effects of an intervention; and 5) provide prognostic information [1-7]. Furthermore, CPET is a common procedure in research. To enable comparison of CPET results to normative data, to compare patient groups and to evaluate the effects of interventions, a strict standardisation of the CPET procedures is required.

There are several statements by the European Respiratory Society (ERS) [8-10], the American Thoracic Society and the American College of Chest Physicians (ATS/ACCP) [11] on exercise testing in respiratory diseases. However, these statements have not focused on the standardisation of CPET, but rather on the value of exercise testing and technical and physiological considerations. In addition, a North American initiative with European input has addressed the harmonisation of terminology in exercise medicine (www. hl7.org/special/Committees/projman/searchableProjectIndex.cfm?action=edit\&ProjectNumber=1246) but has not included standardisation of testing protocols. Thus, the members of this Task Force identified an important missing gap in the current literature concerning CPET: an evidence-based standard on the technical performance of CPET as applied to patients with chronic lung diseases.

Therefore, the overall objective of this ERS Task Force was to standardise CPET procedures for people using CPET as part of clinical and/or research-related investigations (e.g. assessment of the mechanisms/ origin of exercise limitation, evaluation of training programmes and preoperative risk stratification). Thus, the purpose of the document is to provide a comprehensive account of published CPET procedures that will be uniformly applied across different settings, such as research. Specifically, based on a systematic review of the published literature, the Task Force addressed CPET procedures for patients with chronic lung conditions. The Task Force focused on CPET data with a linear work rate increment, either as a ramp protocol or with minute-by-minute increments, since such protocols have been recommended for the assessment of subjects with chronic lung diseases, are widely used in clinical practice [8-10], and are feasible for the assessment of exercise capacity in children (from the age of 10 years), adolescents, adults and elderly people with different chronic lung diseases and various degrees of pulmonary impairment. The Task Force did not include protocols with longer stages, or with an exponentially increasing work rate since such protocols are not as commonly used and/or do not allow assessment of exercise limitations in detail. Furthermore, such protocols have their limitations with respect to important outcomes related to

Affiliations: ${ }^{1}$ Dept of Epidemiology, Biostatistics and Prevention Institute, University of Zurich, Zurich, Switzerland. ${ }^{2}$ Epidemiology, Biostatistics and Prevention Institute, Division of Occupational and Environmental Medicine, University of Zurich and University Hospital Zurich, Zurich, Switzerland. ${ }^{3}$ Lane Fox Respiratory Service, St Thomas' Hospital, Guy's and St Thomas' NHS Foundation Trust, London, UK. 'First Dept of Respiratory Medicine, National \& Kapodistrian University of Athens, Athens, Greece. ${ }^{5}$ Faculty of Kinesiology and Rehabilitation Sciences, Division of Respiratory Rehabilitation, Dept of Rehabilitation Sciences KU Leuven, University Hospital Leuven, Leuven, Belgium. 'Unidade de Fisiologia Pulmonar, Hospital de Clínicas de Porto Alegre, Universidade Federal do Rio Grande do Sul, Porto Alegre, Brazil. ${ }^{7}$ Dept of Paediatric Respiratory and Sleep Medicine, Royal Hospital for Sick Children, Edinburgh, UK. ${ }^{8}$ Paediatric Dept, 424 General Military Hospital, Thessaloniki, Greece. ${ }^{\circ} E L E G I$ Colt Laboratory, MRC Centre for Inflammation Research, The Queen `s Medical Research Institute, University of Edinburgh, Edinburgh, UK. ${ }^{10}$ Respiratory Medicine Dept, Royal Infirmary of Edinburgh, Edinburgh, UK. ${ }^{11}$ HP2 Laboratory, Grenoble Alpes University, INSERM, Grenoble, France. ${ }^{12}$ Hellenic Cystic Fibrosis Association, Athens, Greece. ${ }^{13}$ European Lung Foundation, Sheffield, UK. ${ }^{14}$ Institute of Social and Preventive Medicine, University of Bern, Bern, Switzerland. ${ }^{15}$ Respiratory Rehabilitation and Respiratory Division, University Hospital Leuven, Leuven, Belgium. ${ }^{16}$ REVAL - Rehabilitation Research Center, BIOMED - Biomedical Research Institute, Faculty of Rehabilitation Sciences, Hasselt University, Diepenbeek, Belgium. ${ }^{17}$ Depat of Research and Education, CIRO+, Centre of Expertise for Chronic Organ Failure, Horn, The Netherlands. ${ }^{18}$ Dept of Respiratory Medicine, Maastricht University Medical Centre, NUTRIM School of Nutrition and Translational Research in Metabolism, Maastricht, The Netherlands. ${ }^{19}$ Dept of Physical Therapy, Faculty of Associated Medical Sciences, Chiang Mai University, Chiang Mai, Thailand. ${ }^{20}$ Sorbonne Université, INSERM, UMRS1158 Neurophysiologie respiratoire Expérimentale et clinique, Paris, France. ${ }^{21}$ AP-HP, Groupe Hospitalier PitiéSalpêtrière Charles Foix, Service des Explorations Fonctionnelles de la Respiration, de l'Exercice et de la Dyspnée du Département "R3S", Paris, France. ${ }^{22}$ Division of Paediatric Pulmonology and Allergology, Dept of Paediatrics and Adolescent Medicine, Medical University of Graz, Graz, Austria. ${ }^{23}$ Dept of Rehabilitation Sciences, KU Leuven, Leuven, Belgium. ${ }^{24}$ Pulmonary Rehabilitation, University Hospital Gasthuisberg, Leuven, Belgium. ${ }^{25}$ Laboratory of Clinical Exercise Physiology \& Respiratory Investigation Unit, Kingston Health Science Center, Queen's University, Kingston, ON, Canada. ${ }^{26}$ Rehabilitation Clinical Trials Center, Los Angeles Biomedical Research Institute at Harbor-UCLA Medical Center, Torrance, CA, USA. ${ }^{27}$ Dept of Sport, Exercise and Rehabilitation, Faculty of Health and Life Sciences, Northumbria University, Newcastle, UK. ${ }^{28}$ Paediatric Dept, University Hospital Würzburg, Würzburg, Germany. ${ }^{29}$ Task Force Co-Chair.

Correspondence: Helge Hebestreit, Universitäts-Kinderklinik, Member of ERN-LUNG, Josef-Schneider-Str. 2, 97080 Würzburg, Germany. E-mail: hebestreitवuni-wuerzburg.de 
exercise increments as the anaerobic threshold (AT, determined from breath-by-breath gas exchange measurements or blood lactate measurements) [12].

The ERS Task Force was formed in October 2016, composed primarily of experts from the ERS Rehabilitation and Chronic Care Group, the Clinical Respiratory Physiology, Exercise and Functional Imaging group, the Paediatric Assembly, the Physiotherapists Group, and an ERS methodologist. Task Force members were clinical scientists and (exercise) physiologists with experience in CPET in a broad range of paediatric and adult lung diseases. The Task Force received support from the European Lung Foundation (ELF) throughout the project.

\section{Methodology}

First, a short Delphi questionnaire was completed by the Task Force members in November 2016 to set the search terms and rules for the literature review in January 2017. Data extraction was completed in November 2017. Between November 2017 and January 2018, a patients' survey was performed with the help of the ELF. Thereafter, the manuscript was drafted. Four Task Force meetings were organised in June 2017, September 2017, February 2018 and September 2018. A detailed description of timeline and processes is available in table $\mathrm{S} 1$.

\section{Literature search \\ Inclusion and exclusion criteria}

The literature search focused on studies reporting on CPET (e.g. testing protocols and work rate increments) in patients with pre-defined lung diseases (table 1). We included studies that used exercise protocols on a cycle ergometer or treadmill based on minute-by-minute or ramp increments of work rate. Studies published in European languages and including at least 15 patients per study were considered for analyses. We excluded studies in which exercise tests were performed: 1) without pulmonary gas exchange analysis; 2) under hypoxic conditions; 3) in an environmental chamber; and/or 4) on a cycle ergometer in (semi-) supine position. Review papers, book chapters and conference proceedings were also excluded.

\section{Search methods}

EMBASE, Medline (Ovid), PubMed (PreMEDLINE only), Scopus, Web of Science and the Cochrane Library were searched by an experienced librarian for all articles published from database inception to 12 January 2017. The detailed search strategy for each database can be found in tables S2-S7.

Selection of studies and data extraction

A video conference was held among abstract reviewers to discuss the content and format of the pre-specified screening spread sheets and to re-evaluate inclusion and exclusion criteria to achieve the best possible standardisation. Nine Task Force members screened abstracts for inclusion criteria. Each of these members screened 50 abstracts for training purposes, and the results were compared to those from Task Force members with experience in conducting systematic reviews (M.A. Puhan, R.A. Rabinovich and

\begin{tabular}{|c|c|c|c|c|c|c|c|}
\hline Lung diseases/lung conditions & Studies $\mathbf{n}$ & 1 -min stages & Ramp & Unclear & 1-min stages & Ramp & Unclear \\
\hline COPD/emphysema & 323 & $162(50.2)$ & $92(28.5)$ & $36(11.1)$ & $27(8.4)$ & $4(1.2)$ & $2(0.6)$ \\
\hline Asthma & 19 & $7(36.8)$ & $6(31.6)$ & $1(5.3)$ & $5(26.3)$ & 0 & 0 \\
\hline Interstitial lung disease & 18 & $5(27.8)$ & 12 (66.7) & $1(5.6)$ & 0 & 0 & 0 \\
\hline Primary ciliary dyskinesia & 1 & $1(100)$ & 0 & 0 & 0 & 0 & 0 \\
\hline Sarcoidosis & 12 & $2(16.7)$ & $6(50.0)$ & 4133.3 & 0 & 0 & 0 \\
\hline Lung cancer & 39 & $9(23.1)$ & $21(53.8)$ & 7 (17.9) & $2(5.1)$ & 0 & 0 \\
\hline Unclear respiratory diagnosis & 3 & $3(100)$ & 0 & 0 & 0 & 0 & 0 \\
\hline $\begin{array}{l}\text { Patients undergoing lung transplant or volume } \\
\text { reduction surgery or other thoracic surgeries }\end{array}$ & 23 & $6(26.1)$ & 10 (43.5) & $4(17.4)$ & $1(4.3)$ & 0 & 2 (8.7) \\
\hline
\end{tabular}

Data are $\mathrm{n}(\%)$ for each disease/disease condition. No data were available for tuberculosis and irradiation of the lung. ${ }^{\#}$ : this group contains a mix of different lung diseases (i.e. restrictive and obstructive), asbestosis and obstructive sleep apnoea-hypopnea syndrome. 
T. Radtke). Disagreement was discussed among the group to reach consensus. In case of disagreement on more than five studies, further abstracts were screened and evaluated among Task Force members. Full-text articles were assessed for studies in which exclusion could not be determined from the abstract.

In a second step, detailed information on study populations, exercise protocols and outcomes was extracted and entered into a standardised form. Due to the large number of included studies, data extraction from full-text publications was performed in disease area subgroups. 15 Task Force members were selected with respect to their research expertise. They received training before and during the data extraction process to ensure standardised procedures. Finally, two members (T. Radtke and S. Crook) checked extracted data for consistency and plausibility. If necessary, full texts were re-evaluated and disagreement was resolved between the members.

\section{Patient survey}

In order to gather experiences on CPET from subjects with different lung diseases, the ELF (in collaboration with the Task Force) conducted an online survey from November 2017 to January 2018. The survey consisted of a mix of open-ended, closed-ended and Likert scale questions and was available in nine languages (English, French, German, Greek, Italian, Polish, Portuguese, Russian, Spanish) and was promoted via the ELF and its affiliated patient organisations and the ERS member network. The English version of the patient survey can be found in the online supplement.

\section{Agreement reached}

Based on the literature review and the patients' perspectives, Task Force members drafted text passages that were then circulated among the Task Force members. Issues important for protocol standardisation and controversial points were discussed during two Task Force meetings. The text was refined until all Task Force members fully agreed with its content.

All Task Force members declared and signed conflict of interest statements at the beginning of the project and updated them at project finalisation or when any new relevant conflicts appeared. Conflicts of interests were managed according to ERS rules.

\section{Results from literature review \\ Literature search/data extraction}

A flow chart of included studies is shown in figure S1. Of 7914 identified studies, 595 studies with 26523 patients were included in the quantitative analysis. Table 1 shows exercise modes and protocols used for CPET according to respiratory diagnoses and conditions. In tables S8 and S9, study and patient characteristics are given from studies using either cycle ergometer or treadmill tests. Reported protocol specifications during CPET and outcomes derived from CPET are given in table 2 and table S10, respectively. Table S11 shows characterisation of exercise protocol increments for various pulmonary diagnoses and table 3 specifies criteria used to determine maximal effort during CPET including suggested estimation equations. In table 4, the Task Force panel suggests outcome values to report in standard clinical CPET. Whenever the literature review could not inform the Task Force on specific questions, we referred to existing guidelines or technical standards [8-11].

TABLE 2 Reported protocol specifications during cardiopulmonary exercise testing

\begin{tabular}{|c|c|c|c|c|c|c|}
\hline \multirow[t]{2}{*}{ Protocol specification } & \multicolumn{3}{|c|}{ Cycle test } & \multicolumn{3}{|c|}{ Treadmill test } \\
\hline & $\mathrm{n}(\%)^{\#}$ & Median (IQR) & $n(\%)^{\pi}$ & $\mathrm{n}(\%)^{\#}$ & Median (IQR) & $n(\%)^{\pi}$ \\
\hline Work rate increments & $487(89.2)$ & & & 45 (91.8) & & \\
\hline Rest phase & $264(48.4)$ & & & $10(20.4)$ & & \\
\hline Rest phase duration $\min ^{+}$ & $229(42.0)$ & $3(3-3)$ & 139 (59) & 7 (14.3) & $3(2-3)$ & $4(57)$ \\
\hline Unloaded phase warm-up & $329(60.3)$ & & & 25 (51.0) & & \\
\hline Unloaded duration $\min ^{+}$ & $314(57.5)$ & $3(2-3)$ & 182 (58) & 19 (38.8) & $3(3-4)$ & $10(53)$ \\
\hline Incremental phase duration & $104(19.0)$ & & & 19 (38.8) & & \\
\hline Recovery & $71(13.0)$ & & & 5 (10.2) & & \\
\hline Recovery duration $\min ^{+}$ & $56(10.3)$ & $3(2-6)$ & $18(32)$ & $4(8.2)$ & $3(2-4)$ & $1(25)$ \\
\hline
\end{tabular}

Data are presented as $\mathrm{n}(\%)$ or median (interquartile range). " : studies reporting test protocol details; $\overbrace{}^{\text {: }}$ studies using test duration supported by the literature review; ${ }^{+}$: percentages are calculated of studies that reported on rest, unloaded and recovery phases. 
TABLE 3 Criteria of maximal effort and prediction equations

\begin{tabular}{|c|c|c|c|c|c|}
\hline & \multirow[t]{2}{*}{ Variable } & \multicolumn{2}{|c|}{ Cycle test } & \multicolumn{2}{|c|}{ Treadmill test } \\
\hline & & Children & Adults & Children & Adults \\
\hline 1 & $V_{\mathrm{O}_{2} \text { peak }}^{\prime}$ & $\begin{array}{c}\text { Change of } V_{\mathrm{O}_{2}}^{\prime}<2 \mathrm{~mL} \cdot \mathrm{min}^{-1} \cdot \mathrm{kg}^{-1} \\
\text { for a } 5-10 \% \text { increase in exercise } \\
\text { intensity or of }<2 \mathrm{sD} \text { of the average } \\
\text { increase in } V^{\prime} \mathrm{O}_{2} \text { during the } \\
\text { preceding stages [13] }\end{array}$ & $\begin{array}{l}\text { Change in } V_{\mathrm{O}_{2}}^{\prime} \text { of } \\
<2.1 \mathrm{~mL} \cdot \mathrm{min}^{-1} \cdot \mathrm{kg}^{-1} \\
\text { between consecutive } \\
\text { stages [14] }\end{array}$ & $\begin{array}{c}\text { Change of } V^{\prime} \mathrm{O}_{2} \\
<2 \mathrm{~mL} \cdot \mathrm{min}^{-1} \cdot \mathrm{kg}^{-1} \text { for a } \\
5-10 \% \text { increase in exercise } \\
\text { intensity or of }<2 \mathrm{sD} \text { of the } \\
\text { average increase in } V^{\prime} \mathrm{o}_{2} \\
\text { during the preceding stages } \\
\text { [13] }\end{array}$ & $\begin{array}{c}\text { Change in } V^{\prime} o_{2} \text { of } \\
<2.1 \mathrm{~mL} \cdot \mathrm{min}^{-1} \cdot \mathrm{kg}^{-1} \\
\text { between consecutive } \\
\text { stages [14] }\end{array}$ \\
\hline 4 & RER & $>1.05[16]$ & $>1.05$ [17] & $>1.05$ [16] & $>1.05$ [17] \\
\hline 5 & $\begin{array}{l}\mathrm{HR}_{\text {peak }} \% \text { pred or } \\
\text { beats. } \mathrm{min}^{-1}\end{array}$ & $\geqslant 195$ bpm [18] & $\begin{array}{l}>100 \% \text { pred [11] } \\
231 \text { men and women } \\
\text { laged } 20-80 \text { years) [19] } \\
\text { Female: } 209-0.86 \times a g e \\
\text { Male: } 207-0.78 \times a g e\end{array}$ & $\geqslant 200$ bpm [18] & $\begin{array}{c}>100 \% \text { pred [11] } \\
3320 \text { healthy men and } \\
\text { women laged 19-89 years) } \\
{[17]} \\
211-0.64 \times a g e\end{array}$ \\
\hline
\end{tabular}




\begin{tabular}{|c|c|c|c|c|c|}
\hline & \multirow[t]{2}{*}{ Variable } & \multicolumn{2}{|c|}{ Cycle test } & \multicolumn{2}{|c|}{ Treadmill test } \\
\hline & & Children & Adults & Children & Adults \\
\hline 6 & Blood lactate & $\begin{array}{l}\text { Usually not informative, since } \\
\text { there is considerable variability } \\
\text { in post-exercise blood lactate in } \\
\text { children (e.g. max values } \\
\text { between } 4-13 \mathrm{mmol} \cdot \mathrm{L}^{-1} \text { in } \\
11-13 \text {-year old children] [26] } \\
\text { Minimum post-exercise cut-off } \\
\text { values to be validated [27] }\end{array}$ & $\begin{array}{c}\text { Post-exercise blood } \\
\text { lactate levels } \\
\geqslant 8 \mathrm{mmol} \cdot \mathrm{L}^{-1}[28,29]\end{array}$ & $\begin{array}{l}\text { Usually not informative, since } \\
\text { there is considerable } \\
\text { variability in post-exercise } \\
\text { blood lactate in children le.g. } \\
\text { max values between } \\
4-13 \mathrm{mmol} \cdot \mathrm{L}^{-1} \text { in } 11-13 \text {-year } \\
\text { old children] [26] } \\
\text { Minimum post-exercise } \\
\text { cut-off values to be validated } \\
\text { [27] }\end{array}$ & $\begin{array}{c}\text { Post-exercise blood } \\
\text { lactate levels } \\
\geqslant 8 \mathrm{mmol} \cdot \mathrm{L}^{-1}[28,29]\end{array}$ \\
\hline
\end{tabular}

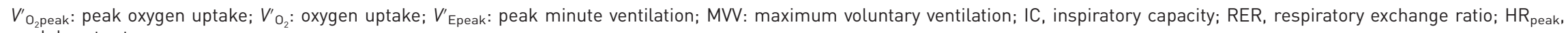
peak heart rate. 
TABLE 4 Outcome values to report in standard clinical cardiopulmonary exercise testing as employed by the Task Force members

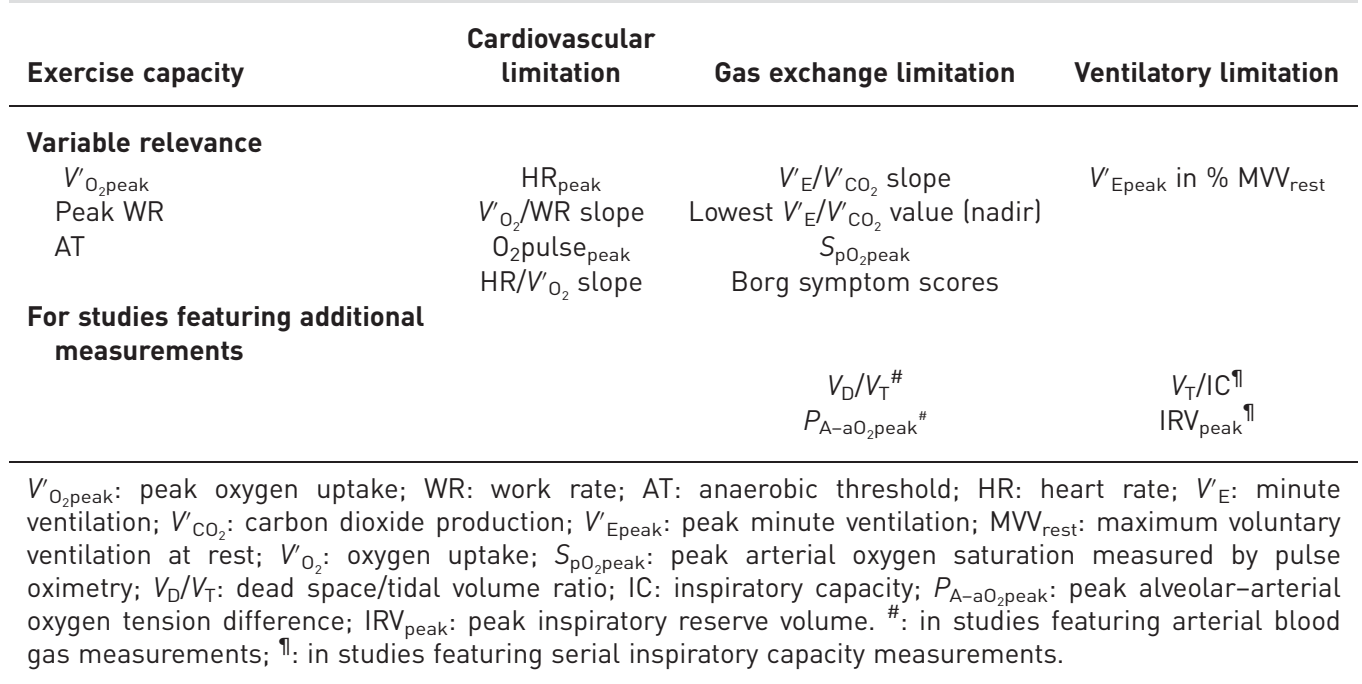

\section{Exercise testing}

Indications/contraindications for exercise testing

CPET is useful in a wide spectrum of clinical practice and a valuable tool in clinical decision making in lung disease $[8,9,11,30]$. In general, CPET is used in lung disease for establishing a diagnosis, determining prognosis, selecting treatment, assessing response to treatment, and follow-up [4]. Indications for CPET in chronic lung disease include the following. 1) Evaluation of "out-of-proportion" (to resting functional impairment) exertional dyspnoea and limited exercise tolerance [3]. 2) Preoperative evaluation (e.g. lung resection surgery, surgical and bronchoscopic lung volume reduction, lung and heart-lung transplantation) [5]. 3) Indication for and prescription of pulmonary rehabilitation [6]. 4) Functional/ disability, prognostic and response to treatment evaluation in subjects with COPD, pulmonary arterial hypertension, pulmonary vascular disease, interstitial lung disease or cystic fibrosis (CF) [1, 2, 31-34]. 5) Assessment of exercise-induced desaturation and underlying mechanisms [35].

CPET is a relatively safe procedure [36] with a rate of death for patients between two and five per 100000 clinical exercise tests [11]. However, potential risks of CPET have to be weighed against the information expected from the test. Contraindications include the following [11, 37].

Absolute contraindications: 1) Uncontrolled cardiovascular conditions (acute myocardial infarction, left main coronary stenosis or its equivalent, unstable angina, moderate or severe stenotic valvular heart disease, symptomatic uncontrolled arrhythmias including atrial fibrillation with uncontrolled ventricular rate, severe untreated arterial hypertension, syncope tachyarrhythmias/bradyarrhythmias, active endocarditis, high-degree atrioventricular block, acute myocarditis or pericarditis, hypertrophic cardiomyopathy, uncontrolled heart failure, suspected dissecting aneurysm, deep venous thrombosis of lower limbs). 2) Uncontrolled respiratory conditions (pulmonary embolism, pulmonary oedema, severe pulmonary arterial hypertension, uncontrolled asthma). 3) Uncontrolled non-cardiorespiratory conditions affecting or aggravated by exercise (e.g. infection, renal failure, thyrotoxicosis, acute bleeding, electrolyte abnormalities). 4) Advanced or complicated pregnancy.

Relative contraindications: 1) Resting oxygen saturation $\left(S_{\mathrm{pO}_{2}}\right) \leqslant 85 \%$ on room air (consider the use of supplemental oxygen). 2) Orthopaedic impairment that compromises exercise performance. 3) Mental or cognitive impairment leading to inability to cooperate.

Verbal assent to proceed with the test will suffice in most situations, but in some settings, in particular research, written consent may be required for CPET.

\section{Physiological responses during cycling versus treadmill testing}

CPET is typically performed using a cycle ergometer or treadmill, each with its own advantages and limitations. The literature review identified many more publications on CPET using cycle ergometry $(\mathrm{n}=546)$ than treadmill exercise $(\mathrm{n}=49)$ (table 1). Likewise, the number of studied patients is much higher for cycle ergometer $(n=23875)$ (table S8) than treadmill tests $(n=2648)$ (table S9) covering a wide spectrum of lung diseases. Although, walking is a familiar activity for most patients and treadmill exercise 
therefore more closely approximates activities of daily living [38] than cycling, the more frequent use of cycle ergometry is likely related to other advantages of this exercise mode [39]: work rate is easier to quantify and control; the variability of the intensity of the exercise increments when using a fixed protocol on cycle ergometer is easier to determine with patients of different body weights; it allows more convenient intra-test procedures such as blood sampling and blood pressure (BP) monitoring, whilst patients are less likely to fall. Moreover, a cycle ergometer is generally cheaper and requires less space than a treadmill.

Cycling and walking clearly elicit different physiological adaptations [40-42]. In this context, information on direct comparison between cycle and treadmill ergometry is scarce and interpretation complicated due to different linearity in the pattern of $V^{\prime} \mathrm{O}_{2}$ response secondary to different work increments applied in each modality [43]. Despite this shortcoming, it has been documented that treadmill compared to cycle ergometry results in the following. 1) Higher peak oxygen uptake $\left(V^{\prime}{ }_{\mathrm{O}_{2 p a k}}\right)$ in healthy individuals $[44,45]$. This finding is in line with the literature review: in patients with various lung diseases such as COPD, asthma, CF, lung cancer or patients undergoing lung transplant or volume reduction, $V_{\mathrm{O}_{2 \text { peak }}}$ achieved during treadmill was, on average, higher as compared to cycling (tables 1 and 2). In addition, it has been documented that during cycling there is lower peak lactate concentration whilst AT being identified at higher $V_{\mathrm{O}_{2}}^{\prime}$ in patients with COPD [46]. 2) Patients during treadmill exercise can exhibit greater systemic arterial oxygen desaturation [46-48]. 3) Regression slopes for breathlessness as a function of both $V^{\prime} \mathrm{O}_{2}$ and minute ventilation $\left(V_{\mathrm{E}}^{\prime}\right)$ are higher [48]. Finally, it seems that breathlessness (rather than leg discomfort) is the most frequently addressed limiting complaint after incremental walking (shuttle walking) compared with incremental cycling ( $81 \%$ versus $34 \%$ ). Conversely, quadriceps fatigue is greater during cycling and is an infrequent feature of incremental walking [49].

These practical, theoretical and physiological characteristics of each modality should guide the practitioner in selecting the ergometer, taking into consideration the reason(s) for which the CPET was requested. In addition, if exercise testing is indicated to provide a prescription for exercise training, it is reasonable to use the same exercise modality that will be used in the training.

\section{Test protocol}

Pre-test procedures

Subjects are usually asked to refrain from eating for at least $2 \mathrm{~h}$ before testing [8] and to avoid strenuous exercise for at least $24 \mathrm{~h}$ before CPET. Subjects are advised to avoid caffeine on the day of the test [8] and smoking for at least $8 \mathrm{~h}$ prior to the test [11] due to the deleterious impact of carboxyhaemoglobin on the oxygen transport capacity. Subjects are asked to wear comfortable clothing and appropriate shoes suitable for exercise. Upon arrival in the laboratory, subject's medical history (with a complete recording of all medications in use), along with physical examination and recording of vital signs (heart rate (HR), BP, $\mathrm{S}_{\mathrm{pO}_{2}}$ and body temperature) is performed to determine any contraindication to exercise testing. In subjects with diabetes mellitus, it is usually advised to measure blood glucose before exercise to reduce the risk of hypoglycaemia [50]. In case of severe hypoglycaemia, defined as a blood glucose $\leqslant 2.8 \mathrm{mmol} \cdot \mathrm{L}^{-1}$ or $50 \mathrm{mg} \cdot \mathrm{dL}^{-1}$, or a hyperglycaemic event in the previous $24 \mathrm{~h}$ requiring assistance, exercise testing is contraindicated [50]. Subjects currently taking bronchodilators are asked to administer their usual bronchodilator treatment at least $10 \mathrm{~min}$ prior to CPET [51]. Attention is paid to the temperature in the exercise laboratory $\left(20-22^{\circ} \mathrm{C}\right)$ [52], as cold air may foster bronchospasm in susceptible individuals.

It is important to make clear to the subjects that sensations during the test such as shortness of breath and a heightened sensation of leg effort are to be expected and their quantification and differentiation will be important for test interpretation. Subjects are informed that the CPET will be terminated at any time they choose to stop. However, it is emphasised that data collected throughout the test will be highly valuable if the participant works to near maximal capacity by the end of the CPET. Subjects are then introduced to a perceived exertion scale and carefully "anchored" to the extremities of the scale as pertaining to both "shortness of breath" and "leg effort". The 0-10 modified Borg scale is the most widely used in clinical practice wherein " 0 " means no dyspnoea at all and "10" the worst shortness of breath ever felt $[53,54]$. The following is an example of general instructions, used by the Task Force members: "During every stage of exercise, we are going to ask you about the intensity of your breathlessness and leg discomfort at that point in time. As you should avoid speaking during the test, you will use a finger of your hand to point which number between 0 and 10 best reflects the intensity of each of these sensations". It is good practice for the technician or physician to say the number out loud to confirm the selected number.

Most commercially available CPET systems now allow measurements of exertional inspiratory capacity (IC) to track the behaviour of operational lung volumes (especially relevant in obstructive lung diseases) [55]. For the identification of mechanisms of exertional dyspnoea, measurements of IC are necessary to quantify the magnitude of exercise-induced dynamic hyperinflation. If the measurement of IC is planned during 
the test, it is important to explain the correct execution of the manoeuvre to the individual before the test with special emphasis on the importance of fully inflating their lungs. An example for general instructions is given in the online supplement [56].

Spirometry should be performed prior to CPET [57]. If direct measurement of maximal voluntary ventilation (MVV) is to be performed, this also should be performed prior to CPET testing [57]. Whilst direct measurement of MVV may be the gold standard, it is highly effort dependent and can be unpleasant to perform with the participant feeling light-headed or dizzy, and in some subjects performance of MVV may induce fatigue. Furthermore, patients often hyperinflate during a voluntary hyperpnoea manoeuvre which can increase their work of breathing relative to the same ventilation during the exercise test $[58,59]$. Finally, after an MVV manoeuvre, in the experience of Task Force members, $15 \mathrm{~min}$ are required to recover to baseline with respect to ventilation and gas exchange.

Calibration of the flow sensor and the gas analysers of the metabolic cart are usually performed just prior to starting the CPET (see below for more information).

\section{Resting phase}

A brief resting period before initiation of exercise allows the patient to familiarise with the testing apparatus (facemask or mouthpiece, ergometer, ECG probes, etc.) and perform baseline measurements of $S_{\mathrm{pO}_{2}}$, arterial blood sampling (in selected cases only), ECG, BP, $V_{\mathrm{E}}^{\prime}$ and gas exchange variables. Resting measurements, especially respiratory exchange ratio (RER), can be helpful in identifying patients hyperventilating before exercise and can be compared with data obtained during maximal effort. The literature review showed that the duration of a resting phase was only reported in $42.0 \%$ and $14.3 \%$ for cycle and treadmill tests, respectively. Of publications selected during the review process, the most common resting phase duration being reported was $3 \mathrm{~min}$ (table 2). This duration is in line with previous guidelines and statements $[8,11]$. Most Task Force members keep the duration of the resting phase up to $3 \mathrm{~min}$ to check that the system is working properly or as long as it is required to obtain accurate and stable resting measurements (e.g. RER $<1.0, V_{\mathrm{O}_{2}}^{\prime}$ approximately $3.5 \mathrm{~mL} \cdot \mathrm{kg}^{-1} \cdot \mathrm{min}^{-1}$ ) [52]. This is in agreement with the results from the literature review (table 2). It is possible not to take measurements during the entire resting phase but only during the final minute or as long as it takes to reach baseline values. When tests are conducted with oxygen supplementation, a longer resting period may be needed to allow for equilibration of higher fraction of inspired oxygen $\left(F_{\mathrm{IO}_{2}}\right)$ in the alveolar space. Patients can be asked to take a few deep breaths to expedite this process. If the measurement of IC is planned during the test, at least three reproducible IC manoeuvres are performed during the resting phase [60].

\section{Unloaded phase}

An unloaded phase follows the resting phase. This phase is important in assessing baseline $V_{\mathrm{O}_{2}}^{\prime}$ and ventilation associated with vertical leg movements and is necessary for the patient to familiarise themselves with exercise on the treadmill/cycle ergometer and to warm-up adequately. Most commercially available cycle ergometers cannot provide a true minimum load of $0 \mathrm{~W}$. Preferably, the minimum work rate on the cycle ergometer does not exceed $10 \mathrm{~W}$ during the "unloaded" phase, and this work rate should be stated in the test report. A three-minute duration for the unloaded phase has previously been recommended $[8,9,11]$ and is in line with the literature review results (table 2). In patients with severe lung disease, the unloaded phase may be shorter (but not $<2 \mathrm{~min}$ ) in order to perform the incremental exercise phase before inducing significant amount of fatigue. If a treadmill is used, the lowest speed may be chosen for baseline measurements, e.g. $1.0-1.6 \mathrm{~km} \cdot \mathrm{h}^{-1}$ [11].

\section{Incremental exercise phase}

After resting and unloaded phases, the incremental exercise phase starts. An incremental exercise protocol adapted to the patient's characteristics is used in order to allow adequate evaluation and follow-up. Ramp and uniform minute-by-minute incremental protocols are both acceptable and provide similar outcomes, when total duration of the incremental phase is the same [61, 62]. According to our literature review, minute-by-minute protocols were used more frequently than ramp protocols for both cycle ergometer (272 versus 216 studies) and treadmill (38 versus 7 studies) exercise testing with 58 (cycle) and 4 (treadmill) studies not reporting the protocol specifications (table 1). While there was a clear preference for minute-by-minute cycle ergometer testing protocols in studies on COPD/emphysema and $\mathrm{CF}$, a protocol preference was less clear for other respiratory conditions. Previous work suggests no differences in physiological parameters (e.g. maximal $\mathrm{HR}, V_{\mathrm{O}_{2 \text { peak }}}$ and $V_{\mathrm{E}_{\text {peak }}}^{\prime}$ ) between minute-by-minute and ramp exercise testing protocols, in both health and disease [11, 63, 64].

The selected work rate increment allows the duration of the incremental exercise phase to be approximately $10 \mathrm{~min}$, typically ranging between 8 and $12 \mathrm{~min}$ [8]. Choice of the rate of increment 
depends on underlying disease, age, sex, body size, pulmonary function, physical activity, fitness and possible other factors such as genetics and medication [65-67]. The incremental phase is designed to last about $10 \mathrm{~min}$, somewhat shorter or longer tests may also provide useful information. However, the work rate- $V^{\prime} \mathrm{O}_{2}$ relationship may not be linear in tests lasting $<6 \mathrm{~min}$, and may not be useful to assess submaximal data such as AT [68]. Most importantly, on repeated testing, the same work rate increment is used. If the measurement of IC is planned during the test, the IC manoeuvres are preferably performed every 2 min and, if possible, at the end of exercise [60].

\section{Cycle ergometry protocols}

Some studies have provided work rate increment estimation for cycle ergometry based on different variables for patients with COPD [65], CF [66], asbestosis, silicosis and asthma [67]. Other, more general recommendations for the selection of work rate, increments are based on estimated $V_{\mathrm{O}_{2 \text { peak }}}$ and $V^{\prime} \mathrm{O}_{2}$ during unloaded pedalling [8]. An approach to establish work rate increments similar to that of Wasserman et al. [12] was adopted by our Task Force. Table 1 and table S11 provide information on the work rate increments used and the median peak work rate and $V^{\prime} \mathrm{O}_{2 \text { peak }}$ measured, as well as the reported protocol specifications (i.e. median time of each phase, etc.) in the studies included in the literature review which can contribute to the determination of work rate increments in individual patients. Very few studies reported test-retest reliability of $V_{\mathrm{O}_{2 p e a k}}$ and peak work rate (table S12).

Based on data available in the literature, we estimated median $V^{\prime}{ }_{\mathrm{O}_{2 \text { peak }}}$ per kg body weight for the populations of each pulmonary disease/disease condition (table S13). The patient's $V_{\mathrm{O}_{2}}^{\prime}$ at unloaded pedalling (in $\left.\mathrm{mL} \cdot \mathrm{min}^{-1}\right)$ can be estimated from the patient's body weight according to the equation $(150+(6 \times$ bodyweight, $\mathrm{kg})$ ) [12]. Assuming an average $V_{\mathrm{O}_{2}}^{\prime}$ increase of $10 \mathrm{~mL} \cdot \mathrm{W}^{-1} \cdot \mathrm{min}^{-1}$ and an assumption regarding the kinetic delay between increase in exercise intensity and $V^{\prime} \mathrm{O}_{2}$ response [11], work rate increments (in W), in order to obtain a $\sim 10$-min incremental exercise phase duration, are calculated as follows:

$$
\begin{gathered}
\text { Cycling work rate increment (Watts per min) }=\left(\text { estimated } V^{\prime} \mathrm{O}_{2 \text { peak }} \text {-estimated } V^{\prime} \mathrm{O}_{2}\right. \\
\text { unloaded } \left.\left(\mathrm{mL} \cdot \mathrm{min}^{-1}\right)\right) / 92.5
\end{gathered}
$$

For example, given a patient with CF, $172 \mathrm{~cm}$ tall and weighing $60 \mathrm{~kg}$, his estimated $V^{\prime} \mathrm{O}_{2}$ during unloaded pedalling is $(150+(6 \times 60))=510 \mathrm{~mL} \cdot \mathrm{min}^{-1}$. According to table $\mathrm{S} 13$ estimated $V^{\prime}{ }_{\mathrm{O}_{2 \text { peak }}}$ per $\mathrm{kg}$ body weight for this patient should be $34.4 \mathrm{~mL} \cdot \mathrm{kg}^{-1} \cdot \mathrm{min}^{-1}$, therefore estimated $V_{\mathrm{O}_{2 \text { pak }}}$ for this patient should be $34.4 \mathrm{~mL} \cdot \mathrm{kg}^{-1} \cdot \mathrm{min}^{-1} \times 60 \mathrm{~kg}=2064 \mathrm{~mL} \cdot \mathrm{min}^{-1}$. Hence, for this patient cycling work rate increment can be calculated as follows:

Cycling work rate increment $($ per $\min )=\left(2064 \mathrm{~mL} \cdot \mathrm{min}^{-1}-510 \mathrm{~mL} \cdot \mathrm{min}^{-1}\right) / 92.5 \mathrm{~mL} \cdot \mathrm{W}^{-1}=16.80 \mathrm{~W} \cdot \mathrm{min}^{-1}$

Tables S8 and S9 provide information on weighted median $V^{\prime}{ }_{\mathrm{O}_{2 p a k}}$ per kg body weight for multiple lung diseases indicated for cycle ergometer and treadmill testing. Estimated work rate increments have proved difficult to establish due to the heterogeneity of reported patient data sets $(>300)$ and lung diseases (table S13). Depending on a subject's regular physical activity, fitness, reported dyspnoea in daily life (e.g. Medical Research Council dyspnoea scale) and resting lung function impairment (e.g. forced expiratory volume in $\left.1 \mathrm{~s}\left(\mathrm{FEV}_{1}\right)\right)$, work rate increments for an individual test will need to be adjusted. Based on the current practice of the Task Force members, adjustments of $\pm 5 \mathrm{~W} \cdot \mathrm{min}^{-1}$ are usually sufficient. Nevertheless, validated, easy to use, and disease-specific equations are required and most likely will improve estimation of work rate increment.

Task Force members would only consider repeating a test with a different work rate increment if data from the test are insufficient to answer the key clinical or research questions that were posed when indicating the test.

\section{Treadmill protocols}

Various well-established treadmill protocols exist for CPET testing including those of BRUCE et al. [69], NAGLE et al. [70] and the modified protocol of NAUGHTON et al. [71], during which treadmill speed and/or slope is increased over time. However, none of these protocols, including their modifications, allows a linear increase of work rate as a ramp or minute-by-minute increment. However, ramp protocols with linear increase in work rate have been developed for treadmill testing in healthy adults [43, 60]. PorsZASZ et al. [43] developed an algorithm using body weight to produce a linear increase in work rate on the treadmill that more closely mimics the linear increase in metabolic rate seen with cycle ergometry. The algorithm of PORSZASZ et al. [43] has been adapted to assess patients with diseases such as COPD [47, 72]. For other conditions, treadmill work rate increments have not been published. Due to the low number of studies using treadmill protocols identified in this review, disease-specific estimation of work rate increments was felt to be inadequate. 


\section{Recovery phase}

After the end of the incremental exercise phase (i.e. after reaching the patient's limit of tolerance), all Task Force members employ a recovery period of at least 2-3 min with unloaded pedalling at a reduced cadence of $\sim 30$ revolutions $\mathrm{min}^{-1}$ or very slow walking. A prolonged recovery period may be indicated for individual patients. The recovery phase is useful for safety reasons and may also be of interest for patients to demonstrate improvements in exercise recovery time after pulmonary rehabilitation and exercise training programmes. During this period, cardiovascular monitoring (ECG, BP, $S_{\mathrm{PO}_{2}}$, etc.) is typically performed. Since delayed post-exercise HR recovery is frequently observed in lung disease patients and associated with dynamic hyperinflation [73] and poor prognosis [74, 75], HR recordings may offer additional useful information. Furthermore, assessment of perceived exertion, dyspnoea and leg fatigue is done using standardised scales suggested by Borg et al. [76, 77] and GIFT [78]. Gas exchange measurements can be terminated and the mask/mouthpiece removed in order to make the patient more comfortable (unless these measurements are required for specific evaluations during the recovery phase), as was done in patients suffering from hyperventilation syndrome [79]. In addition, IC manoeuvres may be performed to assess the rate of recovery of dynamic hyperinflation, but this is mainly done for research purposes.

A flow chart describing the usual practice by Task Force members on the different test protocol phases is given in figure 1 .

$\mathrm{V}^{\prime} \mathrm{O}_{2 \text { peak }}$ verification phase

A supramaximal verification test is a high-intensity, constant load test performed after CPET [80, 81]. Work rate selected for this task is $>100 \%$ peak power output achieved during CPET. Details on the methodology of supramaximal verification of $V^{\prime} \mathrm{O}_{2}$ are provided in the online supplement. Due to paucity of data in lung disease patients [80-82], the majority of Task Force members abstain from a supramaximal verification test as part of routine clinical CPET. The test may, however, be useful for research questions and in cases with suspected invalid peak data during CPET.

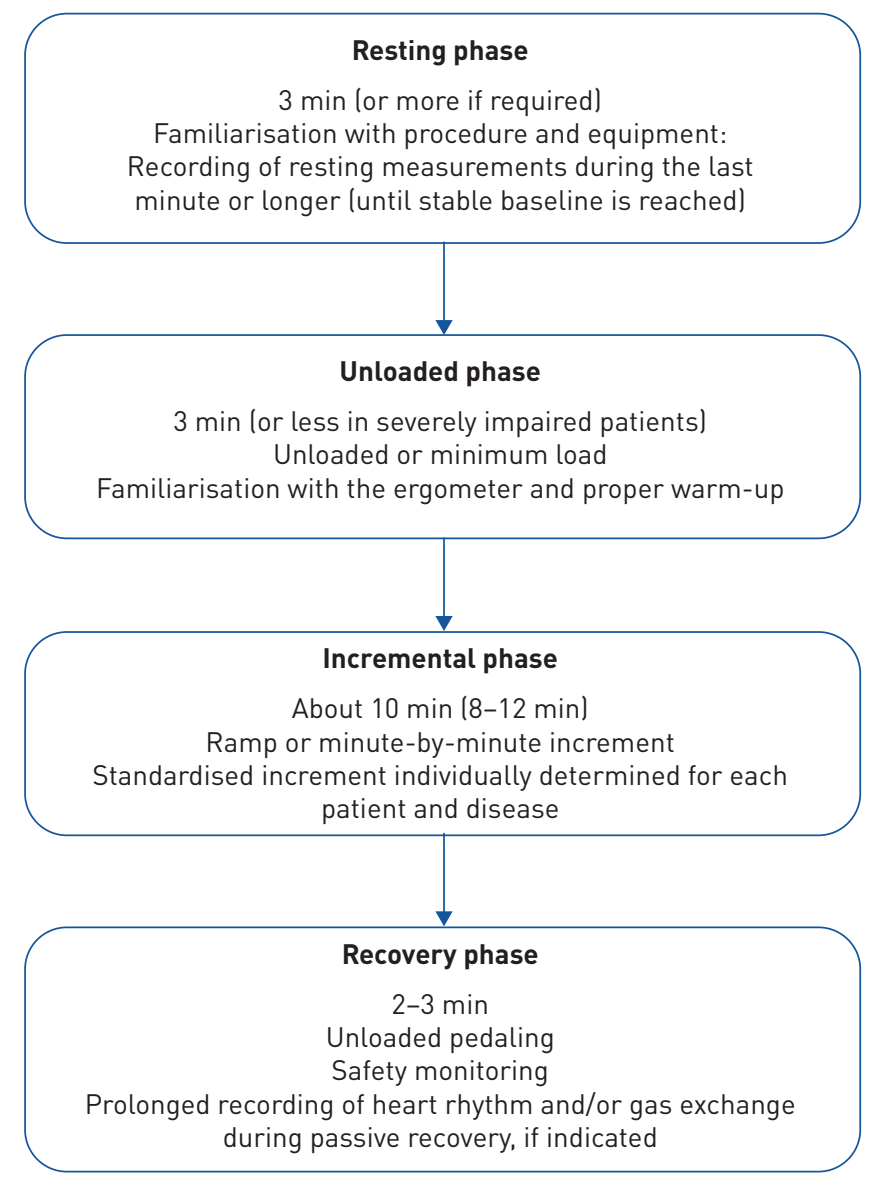

FIGURE 1 Flow chart describing the usual practice by Task Force members on different protocol phases and specifications during cardiopulmonary exercise testing. It is not intended as a recommendation for clinical practice. 


\section{Technical considerations}

\section{Cycle ergometer}

Mechanically braked cycling requires maintenance of a specific cycling rate to achieve the specified work rate [35]. In contrast, performed work rates during electronically braked cycling are directly quantified and can be computer controlled, leading to work rate being maintained over a wide range of pedalling rates (40-70 revolutions per minute) [11]. This allows work rate to be incremented automatically and continuously (e.g. ramp protocol) [11]. According to the American Heart Association (AHA), electronically braked ergometers have become the standard in clinical testing; but are more expensive and less portable [52]. Cycle ergometers used in patient testing are medical devices and need to fulfil all respective requirements. A cycle ergometer needs to be capable of adjusting work rate in increments either automatically or manually [52]. For the most debilitated patients with lung diseases, it is important to use a cycle ergometer that allows very low (near zero) work rates during unloaded pedalling, so that patients do not have to pedal against flywheel inertia [11]. In addition, the cycle ergometer must include adjustable handlebars and seat (and crank arms in smaller children), allowing the knee to be slightly flexed when the pedal is at its lowest position [83]. Meters, dials or digital displays should be appropriately sized and placed for easy reading [52]. It is advised that adaptable pedal grips (i.e. to fix the patients' shoes on the pedal) are employed to optimise performance and increase patients safety [52]. Regular calibration of the equipment is necessary following the manufacturer's specifications. This usually includes measurements of crank arm torque at various speeds using a certified instrument. If not required more often by local regulations or manufacturer, verification of calibration should be done annually and after each service/repair of the ergometer [11]. Further information on calibration procedures and quality control can be found elsewhere [84].

\section{Treadmill}

Treadmills for CPET should be electrically driven, are medical devices, and therefore must meet all safety standards [52]. Optimally, these allow the use of a wide range of individualised incremental and/or ramp protocols. Specifically, in patients with lung diseases, it is essential that the treadmill offers very slow walking speed (e.g. $1 \mathrm{~km} \cdot \mathrm{h}^{-1}$ ) and allows inclination of choice. The AHA proposes that a suitable treadmill should minimally be $127 \mathrm{~cm}$ in length and $41 \mathrm{~cm}$ in width and should accommodate body weights up to $150 \mathrm{~kg}$ [52]. It is advised that a padded front rail and at least one side rail are present to optimise patient safety, but minimal or (preferably) no handrail support needs to be encouraged as it influences the metabolic cost of locomotion [68]. An emergency stop button is always visible and directly accessible to staff and patient. As in cycle ergometry, verification of speed and inclination readings of the treadmill should be done at least annually [11].

\section{Mouthpiece versus facemask}

Subjects can be interfaced to the metabolic cart system via a bite-block mouthpiece, which is combined with a nose clip and often stabilised by headgear or via a facemask assembly made of pliable rubber that covers the area of the nose and mouth. Although the mouthpiece/nose clip combination is the gold standard for measuring respiratory parameters during an incremental exercise test, it can be uncomfortable for some participants as it makes swallowing difficult, and promotes dry mouth, and/or throat irritations [85]. In contrast, a facemask assembly provides an alternative to mouthpiece/nose clip that allows oral and often nasal breathing, as well as swallowing [86]. However, a major concern is that, with a facemask, it may not be possible to get a tight fit to the face, associated with relevant leakage particularly at high exercise intensities, thus yielding inaccurate respiratory measurements. Furthermore, the dead space $\left(V_{\mathrm{D}}\right)$ is usually larger when using a facemask compared to a mouth piece/nose clip. Past studies in athletes and lung disease patients have reported lower values of $V_{\mathrm{O}_{2}}^{\prime}$ (ranging from $5.8 \%$ to 24\%) during facemask breathing as compared to mouthpiece/nose clip [87-90]. In addition, parameters such as $V_{\mathrm{E}}^{\prime}$, tidal volume $\left(V_{\mathrm{T}}\right)$, carbon dioxide output $\left(V^{\prime} \mathrm{CO}_{2}\right)$ and respiratory rate have been reported to be changed when a facemask was used $[85,86,89,90]$. Nevertheless, current facemasks are lighter in weight, have improved contact surface area to prevent leakage, and create a more comfortable fit as compared to previous models. Indeed, a recent study in healthy individuals did not report any differences either in gas exchange or breathing patterns between the two different breathing apparatus during both sub-maximal and maximal levels of exercise [91]. In any case, $V_{\mathrm{D}}$ of the system used needs to be fed into the metabolic cart/analysing software.

From a patient's perspective, the survey respondents showed no clear preference between mouthpiece/nose clip and facemask assembly use among 34 (14\%) participants who had experience of using both (figures S2-S4). Of these, 12 participants preferred a mouthpiece, 10 preferred a facemask and 12 had no preference. Based on the evidence and patient input, both approaches are, therefore, acceptable. Some additional concerns outlined by respondents who had used both assemblies to those outlined above were that use of the mouthpiece gave pain in the jaw and palette, a sensation of choking and saliva dripping 
from the mouth can be embarrassing for patients. Additional concerns from using a facemask were that it gave a feeling of tightness/pressure, panic, fear of suffocation and pain from wearing the mask. It would seem that use of each assembly is likely to be a personal preference and therefore, where possible, a choice should be offered to patients following discussion of the advantages and disadvantages of each.

Pulse oximetry

Pulse oximeters rely on the differential transmission of certain light wavelengths by reduced and oxygenated haemoglobin [92] to estimate arterial oxyhaemoglobin saturation. Pulse oximetry is used to monitor $S_{\mathrm{pO}_{2}}$ during CPET, which gives a general oxygenation estimate providing a trend during the test $[11,68]$. However, it is reported as an outcome in only around $30 \%$ of published studies using CPET as part of patient assessment (table S10).

A $\geqslant 5 \%$ fall in $S_{\mathrm{pO}_{2}}$ during CPET suggests exercise-induced hypoxaemia [11]. In some laboratories, falls of $\mathrm{S}_{\mathrm{pO}_{2}}$ below $85 \%$ or $80 \%$ constitute an indication to stop the test. Pulse oximeters' accuracy rates during exercise are reasonably good (95\% CI 4-5\%) when compared with direct measurement in arterial blood oxygenation [93]. Wider confidence limits, however, have been reported, particularly when $S_{\mathrm{pO}_{2}}$ falls below $88 \%$ [94] with even further decrease in reliability in populations with dark skin [95, 96]. Most common inaccuracies in the measurements are due to poor capillary perfusion during exercise (mainly in cardiovascular diseases) and motion artefacts [97] providing inaccurately lower levels of $S_{\mathrm{pO}_{2}}$. An inaccurate pulse rate reading from the oximeter can sometimes identify these inaccuracies, but this is not always the case. Some authors have reported overestimation of $S_{\mathrm{pO}_{2}}$ measured with pulse oximetry [98-100].

Moreover, sensor placement (i.e. finger, earlobe, nose, forehead) may also affect the stability and reliability of $S_{\mathrm{pO}_{2}}$ recordings. For example, in healthy people and those with cardiovascular disease, forehead sensors have better precision compared to finger sensors under various testing conditions (i.e. normoxia, hypoxia and hyperoxia) [101]. Hand motion has been shown to reduce signal quality during finger $S_{\mathrm{pO}_{2}}$ recordings [102] suggesting use of ear or forehead sensors during cycling or treadmill exercise to be more appropriate. In patients with digital clubbing, for example in CF, finger sensors underestimate $S_{\mathrm{pO}_{2}}$ readings [103]. Forehead sensors have the advantage that they are less affected by motion and can possibly reduce the measurement error [101].

It should be recognised that pulse oximeters do not detect methaemoglobin or carboxyhaemoglobin and will overestimate oxyhaemoglobin saturation in circumstances where these are elevated [104]. In addition, due to the haemoglobin dissociation curve shape, $S_{\mathrm{pO}_{2}}$ measurements are relatively insensitive to changes in arterial oxygen tension $\left(P_{\mathrm{aO}_{2}}\right)$ at levels corresponding to the plateau of the curve [105] (i.e. $S_{\mathrm{pO}_{2}}$ will remain above $93 \%$ and hardly change at $P_{\mathrm{aO}}$ values $>70 \mathrm{mmHg}$ ).

\section{Arterial blood gas measurements}

The measurement of arterial blood gas requires the placement of an arterial catheter prior to exercise, preferably into the radial artery [11]. The clinical decision for arterial blood gas measurements during CPET depends on the purpose of the test; e.g. if pulmonary gas exchange abnormalities are to be expected and noninvasive estimation of oxygenation is considered inadequate [11]. Blood samples can be taken at rest, after the unloaded phase, every other minute during the incremental phase and after 2 min of recovery [11].

In general, measurement of $P_{\mathrm{aO}}$ allows the calculation of gas exchange indices such as the alveolar-arterial oxygen tension difference. In this case, arterial blood sampling needs to be done using arterialised capillary blood or from an indwelling arterial catheter at the end of exercise [106]. There is evidence showing that a puncture performed within $20 \mathrm{~s}$ after exercise may capture all episodes of significant hypoxaemia (i.e. $P_{\mathrm{aO}_{2}}<60 \mathrm{mmHg}$ ) [107]. Moreover, arterial or arterialised capillary blood gas analyses have the potential to provide relevant information on arterial carbon dioxide tension, $\mathrm{pH}$, bicarbonate and lactate levels. The measurement of arterial carbon dioxide tension allows the calculation of $V_{\mathrm{D}} / V_{\mathrm{T}}$, a measure of the efficiency of $\mathrm{CO}_{2}$ exchange. $P_{\mathrm{aO}}$ levels obtained from capillary blood are less accurate and should be corrected for the systematic bias between adequately detected disturbances in pulmonary gas exchanges with a high sensitivity and specificity as previously described [108]. Of note, arterialised earlobe blood is not a reliable measurement of $P_{\mathrm{aO}}$ [109].

\section{Heart rate/blood pressure \\ Electrocardiogram: heart rhythm and ischaemia}

Continuous recording of heart rhythm and evaluation of ischaemic changes during exercise and recovery is crucial for safety and diagnostic reasons. The electrocardiograph should meet the specifications set by the AHA [110]. The use of two monitors (one for ECG curves) or a wide monitor that is separated into two screens may facilitate visualisation of ECG curves and detection of arrhythmias during exercise. To detect arrhythmia and/or ischaemia, continuous oscilloscopic monitoring is recommended [52]. 
Additionally, the capacity to deliver a 12-lead printed copy improves interpretation. The Mason-Likar modification of the 12-lead ECG electrode placement has been widely used in the clinical setting; however, it may modify the inferior lead complexes $[111,112]$. Thus, a standard resting 12-lead ECG is obtained before CPET [8, 11]. Silver/silver chloride electrodes are recommended for reducing motion artefacts [52]. Skin preparation (i.e. shave area where the electrode is placed) is extremely important regardless of the type and size of electrodes to achieve high signal quality [52]. Connecting cables between the electrodes and recorder should be light, flexible and properly shielded to further stabilise the electrocardiographic signal [52]. Flexible knit "tube" shirts for stabilising the electrodes and cables are widely available [52]. In the current literature review, peak HR was reported in $49 \%$ of the included studies and peak HR expressed as percentage of predicted normal was reported in $20 \%$ (table S10). In fact, peak HR was the fourth most frequently reported parameter in the included studies. Reporting peak HR in CPET studies is, therefore, advisable.

\section{Blood pressure monitoring}

Manual auscultation is still perceived as the most feasible and accurate method for monitoring BP. Automated BP devices are widely available and many of them have been designed for use during exercise, but these devices are expensive and prone to measurement error due to the effects of (chest) motion at high exercise intensities, especially affecting diastolic BP [113]. If such devices are used, their accuracy should be validated against manual cuff measurements before routine use during CPET; extreme hyper- or hypotensive systolic BP readings should be immediately confirmed by manual auscultation [52] or pulse palpation using the BP system (cuff and pressure readings) which is in place. The exercise test should be terminated if systolic BP falls $>20 \mathrm{mmHg}$ compared to the highest recording during the test and in case of hypertension (systolic BP >250 mmHg; diastolic BP >120 mmHg) [11]. Cuffs of various sizes, including large and paediatric should be available [12]. The cuff should be positioned at the level of the heart and the apparatus should be calibrated [52]. BP is usually monitored every 2 min during CPET. In the current literature review, peak BP was reported in $10 \%$ of the included studies (table S10). While BP is rarely reported in publications, it is almost unequivocally assessed, according to the experience of the Task Force members.

\section{Metabolic carts}

Accurate recording of respiratory volumes and airflow parameters, as well as inspired and expired gases, requires accurate equipment. With the readily available computers and by utilising specific algorithms, it has become practical to compute respired flow, volumes and gas concentrations breath-by-breath [114]. Pneumotachographs [115], mass flow sensor transducers [116] and turbines, among other devices, are widely used in commercially available metabolic carts for sensing volumes and flows at rest and during laboratory-based exercise testing [117]. These types of transducers must comply with the standards that have been established by the ATS and ERS for flow and volume measurements [57]. These standards specify that the devices must have low $V_{\mathrm{D}}$, low resistance to breathing flows and be immune to temperature changes, and to the water vapor or pools of saliva that may accumulate during exercise [57]. For example, pneumotachographs are sensitive to gas composition variation and temperature changes [118] as compared to turbine flow meters, that are lightweight and have low $V_{\mathrm{D}}$. However, pneumotachographs have been considered as more accurate than mass flow sensor transducers [117] With regard to gas analysers, several systems exist, namely gas collection systems, mixing chamber systems, gas-exchange measurement at elevated inspired $\mathrm{O}_{2}$ concentrations and more highly developed devices without employing the Haldane transformation [119]. The advantages and disadvantages of each system have been previously described in detail [84]. A breath-by-breath system employing a discrete $\mathrm{O}_{2}$ analyser using paramagnetic or electro-chemical cell technology and a $\mathrm{CO}_{2}$ analyser using non-dispersive infrared sensor technology or thermal conductivity, as well as mass spectrometry, are suitable for the demands of CPET [11].

For ensuring valid measurements, the examiner has to follow manufacturer-suggested calibration procedures for flow and gas analysers. Both flow sensors and gas analysers tend to drift, therefore calibration procedures immediately preceding each test are recommended. For flow analysers, this includes calibration using standard volume (typically using a certified $3 \mathrm{~L}$ syringe) in at least three flow ranges, i.e. $2,4-6$ and $8 \mathrm{~L} \cdot \mathrm{s}^{-1}$ to assure linearity. Agreement in calculated volumes within $\pm 3 \%$ signifies acceptable performance [84]. The minimum requirements of gas analysers sensors' performance for measurements during room-air breathing are: 1) for $\mathrm{O}_{2}$ sensors a detection range between $0-100 \%$ and for $\mathrm{CO}_{2}$ sensors a detection range between $0-10 \%$; 2) sensors' accuracy of $1 \%$; 3) sensors' response time of $<13 \mathrm{~ms}$; and 4) two calibration points (for each sensor): one in the range of ambient concentration and the other in the range of exhaled concentration [11, 120]. Preferably, two gases with known gas concentrations should be used for calibration rather than only one gas and room air [11]. With the help of a metabolic simulator [121] or testing the same subject (healthy and fit) in at least two or three different submaximal exercise levels 
(physiological calibration) [122] it is possible to regularly perform a global functioning check for assuring correct ventilatory and gas exchange calculations [84]. The pick-up lead for sampling inspiratory and expiratory gas needs to be regularly replaced by a new one every 3-6 months. Between tests, it is also necessary to change the lead to avoid cross infection.

\section{Test with high oxygen concentration}

CPET in the condition of hyperoxia (supplemental oxygen) has several applications such as detection of desaturation during exercise despite oxygen supplementation in patients with intrapulmonary or cardiac right-to left shunt, and response to supplemental oxygen, particularly in patients with COPD [123-125] and, although with limited literature support, in CF and interstitial lung diseases [10]. Hyperoxia, however, can interfere with the performance of the equipment sensors (i.e. oxygen and flow sensors and gas exchange calculation algorithms). Similarly, at high altitude (hypoxic conditions), reduced humidity and barometric pressure as well as extreme temperatures can impose a challenge to the equipment. Supplemental oxygen can alter viscosity of the respired gas (i.e. $11 \%$ greater for $F_{\mathrm{IO}_{2}}=1$ in comparison to $F_{\mathrm{IO}_{2}}=0.21$ ). In regard to flow sensors, pneumotachographs are more sensitive to changes in air viscosity, temperature and humidity than turbine volume transducers [11, 126, 127]. In addition, density also influences Pitot tube flow meters [11] and mass flow meters (that measure mass flow in contrast to flow of a volume of air) [116]. As for gas concentration sensors, not all of the available methods to measure concentrations of gases, particularly $\mathrm{O}_{2}$, are accurate in hyperoxic conditions. Paramagnetic oxygen analysers' ranges might be limited to $\mathrm{F}_{\mathrm{IO}_{2}}$ between 0 and 0.25 [128]. Thus, the technician or physician may check whether the metabolic cart allows recordings of gas exchange variables when the inspired air contains a high fraction of oxygen.

Another important issue to consider is the kinetics response of the analysers that can be lengthened by the increased viscosity of the air in hyperoxic conditions [129].

When intended to use hyperoxic (or hypoxic) conditions, it is mandatory for the equipment to be adequately validated and calibrated for these conditions. Furthermore, calibration gas mixtures should be selected to reflect the concentrations of inspired and expired air expected [84].

Infection control considerations

In a busy cardiopulmonary exercise-testing laboratory with a large heterogeneity of patients and disease entities examined, cross-infection can occur. Therefore, a number of precautions are usually taken and standards applied.

Cross-infection can occur either via direct contact between patients, indirect contact with contaminated surfaces, equipment or healthcare personnel, and inhalation of aerosolised particles or droplets via airborne route, tubing or mouthpieces/facemasks [130]. Direct contact between patients with lung diseases should be minimised in all cases $[131,132]$. Appropriate hand hygiene by healthcare workers should be applied according to established standards [133] and supervised occasionally in order to improve compliance. Sufficient time intervals between tests should be used for surface decontamination and room aeration according to local infection control guidelines [134]. Parts of CPET apparatus that might be contaminated (e.g. tubing and hand grips) should be disinfected according to manufacturer's recommendations [135]. In case of contamination that cannot be dealt with by available means of disinfection, all contaminated parts are replaced.

In patients known to be infectious, additional precautions may be considered. For example, to prevent inhalation of microorganisms, barrier filters may be added in case of exercise testing with mouthpieces [136]. However, the additional $V_{\mathrm{D}}$ of the filter should be considered in the setup of the metabolic cart. Moreover, calibration of the flow-volume sensor with the bacterial filter in place is required to accommodate for the additional resistance. Each patient should be provided with a new or disinfected nose clip. Regardless of whether CPET is performed with the use of facemask or mouthpiece, after every test facemasks and multi-use mouthpieces are disinfected according to manufacturer's and the laboratory standards [135].

\section{Criteria for determining whether a patient reached the limit of exercise tolerance}

Once data are verified as being technically acceptable, it is important to consider whether the limit of exercise tolerance has been reached. In patient populations, this assessment considers subjective (dyspnoea and leg effort scores, impression of the examiner) and objective criteria (peak HR, RER at peak exercise, $V_{\mathrm{E}}^{\prime}$ in percent of MVV, and whether or not a plateau in $V_{\mathrm{O}_{2}}^{\prime}$ was reached near the end of exercise (attainment of $\left.V_{\mathrm{O}_{2 \max }}^{\prime}\right)$ ).

It is important to begin data interpretation by reviewing $V^{\prime}{ }_{\mathrm{O}_{2 p e a k}}$ and peak work rate and compare to reference values that are age, sex and test specific (table 3). A reduced exercise performance may be due 
(solely or in combination) to cardiovascular, ventilatory, gas exchange or musculoskeletal factors, or simply due to lack of physical conditioning and/or obesity. These need to be separated from reduced exercise performance that reflects suboptimal effort.

The quantification of $V^{\prime}{ }_{\mathrm{O}_{2 \max }}$ (typically by using a cycle ergometer or treadmill) requires $V_{\mathrm{O}_{2}}^{\prime}$ to reach a value where, despite further increases in work rate, no further (or relatively small) increases in $V^{\prime} \mathrm{O}_{2}$ occur; thus a plateau is attained. The plateau required to delineate $V^{\prime}{ }_{\mathrm{O}_{2 \max }}$ from $V_{\mathrm{O}_{2 \text { pak }}}^{\prime}$ is often defined by an increase in $V^{\prime} \mathrm{O}_{2}<2.0 \mathrm{~mL} \cdot \mathrm{min}^{-1} \cdot \mathrm{kg}^{-1}$ despite an increase in work rate by $5-10 \%[13,17]$ or, when exercise intensity is increased minute-by-minute, an increase in $V_{\mathrm{O}_{2}}^{\prime}<150 \mathrm{~mL} \cdot \mathrm{min}^{-1}$ during the last work rate compared with the immediately preceding one [8].

Importantly, it should be kept in mind that there is no gold standard for defining a maximal effort. Criteria to delineate a peak effort are as follows (see also table 3) $[9,11]$. 1) A plateau in $V^{\prime} \mathrm{O}_{2}$ is observed. 2) Peak exercise ventilation ( $V_{\text {Epeak }}^{\prime}$ ) exceeds $85 \%$ of directly measured (sprint method) [57] or estimated MVV [137]. Various methods are used to estimate MVV including: forced expiratory volume in $1 \mathrm{~s}$ $\left(\mathrm{FEV}_{1}\right) \times 35[11], \mathrm{FEV}_{1} \times 40[11,138]$, or disease $[139,140]$ or ethnicity specific equations [141]. Since most laboratories use the equation $\mathrm{FEV}_{1} \times 40$ to estimate $\mathrm{MVV}$, this equation seems to be appropriate and is preferentially used by the majority of Task Force members. 3) Evidence of a ventilatory limitation has been pointed out by the development of significant expiratory flow limitation $\left(>40 \%-50 \%\right.$ of $\left.V_{T}^{\prime}\right)$ and/or decrease in IC from baseline $>150 \mathrm{~mL}$ during exercise indicating dynamic lung hyperinflation $[10,15] .4$ ) Maximal RER during exercise exceeding 1.05 is considered an indicator of a maximal effort [16, 17]. An RER $>1.05$ would be supportive of a maximal test across all ages. 5) The patient reaches a HR at or above calculated $\mathrm{HR}_{\max }$ [11]. 6) $V^{\prime} \mathrm{O}_{2 \text { peak }}$ exceeds $V_{\mathrm{O}_{2 \text { peak }}}^{\prime}$ predicted [11]. 7) Peak work rate exceeds peak work rate predicted [11]. 8) Post-exercise blood lactate levels $\geqslant 8 \mathrm{mmol} \cdot \mathrm{L}^{-1}[28,29]$.

Subjective scores of dyspnoea, measured for example using the 10-point Borg dyspnoea scale [77] or a 0-100 Visual Analogue Scale (VAS) [142], and the assessment of the supervising technician or physician also add to the determination of an effort reaching the limit of tolerance. Both the VAS and the Borg Scale have been shown to provide similar scores during CPET, and to be reliable and reproducible over time in healthy subjects and patients with cardiorespiratory diseases undergoing CPET [143, 144]. In general, patients stop exercise at ratings of 5-8 on the modified Borg Scale or 50-80 on the VAS [11, 15, 145]. In the Task Force working and research experience, ratings indicating severe leg discomfort or dyspnoea on a Borg scale (7-10 units) or VAS scale (70-100) or a high score on a similar perceived exertion scale are often used as an indicator for a peak effort.

Figure 2 provides a description of how Task Force members define a maximal test and start to delineate the reason(s) for exercise limitation in those in whom an abnormal exercise response is noted.

\section{Outcomes to report and variables to consider}

The purpose of CPET is to detect exercise intolerance and, if present, define the mechanism(s) contributing to exercise intolerance. A detailed overview of CPET interpretation in different clinical scenarios has been recently provided in the 2018 ERS Monograph on Clinical Exercise Testing [13].

Briefly, two variables are used to detect exercise intolerance (table 4). $V^{\prime}{ }_{\mathrm{O}_{2 p a k}}$ observed during the exercise test can be compared to normal values [15]. However, $V^{\prime}{ }_{\mathrm{O}_{\text {ppak }}}$ has the disadvantage that it is dependent on motivation as well as physiological capacity [11, 15]. The AT, determined from gas exchange or measurements of blood lactate, defines the point at which lactate production exceeds lactate elimination $[146,147]$. It has the advantage of being effort independent but relies on pattern recognition for accurate detection. Gas exchange methods involve determination of the point at which $\mathrm{CO}_{2}$ output accelerates with respect to $\mathrm{O}_{2}$ uptake, a manifestation of bicarbonate buffering of lactate. The exercise intensity at which lactate increases, bicarbonate decreases and $\mathrm{CO}_{2}$ output accelerates have been found to be similar, although modest differences have been detected [148, 149].

Defining cardiovascular limitation to exercise relies on a number of interrelated variables. A low slope or terminal flattening of the $V^{\prime} \mathrm{O}_{2}$ over work rate relationship or flattening of oxygen pulse may be diagnostic [3]. Pulmonary vascular causes of cardiovascular limitation may yield a high $V^{\prime}{ }_{E} / V^{\prime} \mathrm{CO}_{2}$ slope in addition to those abnormalities. Low haemoglobin levels will produce oxygen flow deficiency. Primary cardiovascular limitation may manifest with ECG or BP abnormalities.

Gas exchange limitation to exercise can be $\mathrm{CO}_{2}$-related, $\mathrm{O}_{2}$-related or both. Inefficient $\mathrm{CO}_{2}$ exchange is manifested by high $V_{\mathrm{D}} / V_{\mathrm{T}}$, often signalled by high exercise $V^{\prime}{ }_{\mathrm{E}} / V^{\prime} \mathrm{CO}_{2}$. A recent publication confirmed the specificity of high $V_{\mathrm{E}}^{\prime} / V^{\prime} \mathrm{CO}_{2}$ in defining elevation in $V_{\mathrm{D}} / V_{\mathrm{T}}$ (as opposed to ventilatory control-mediated alteration in arterial carbon dioxide tension) [150]. Inadequate oxygen exchange can be detected by reductions in $P_{\mathrm{aO}_{2}}$ or, less directly, by fall in $\mathrm{S}_{\mathrm{pO}_{2}}$ assessed by pulse oximetry [3]. 


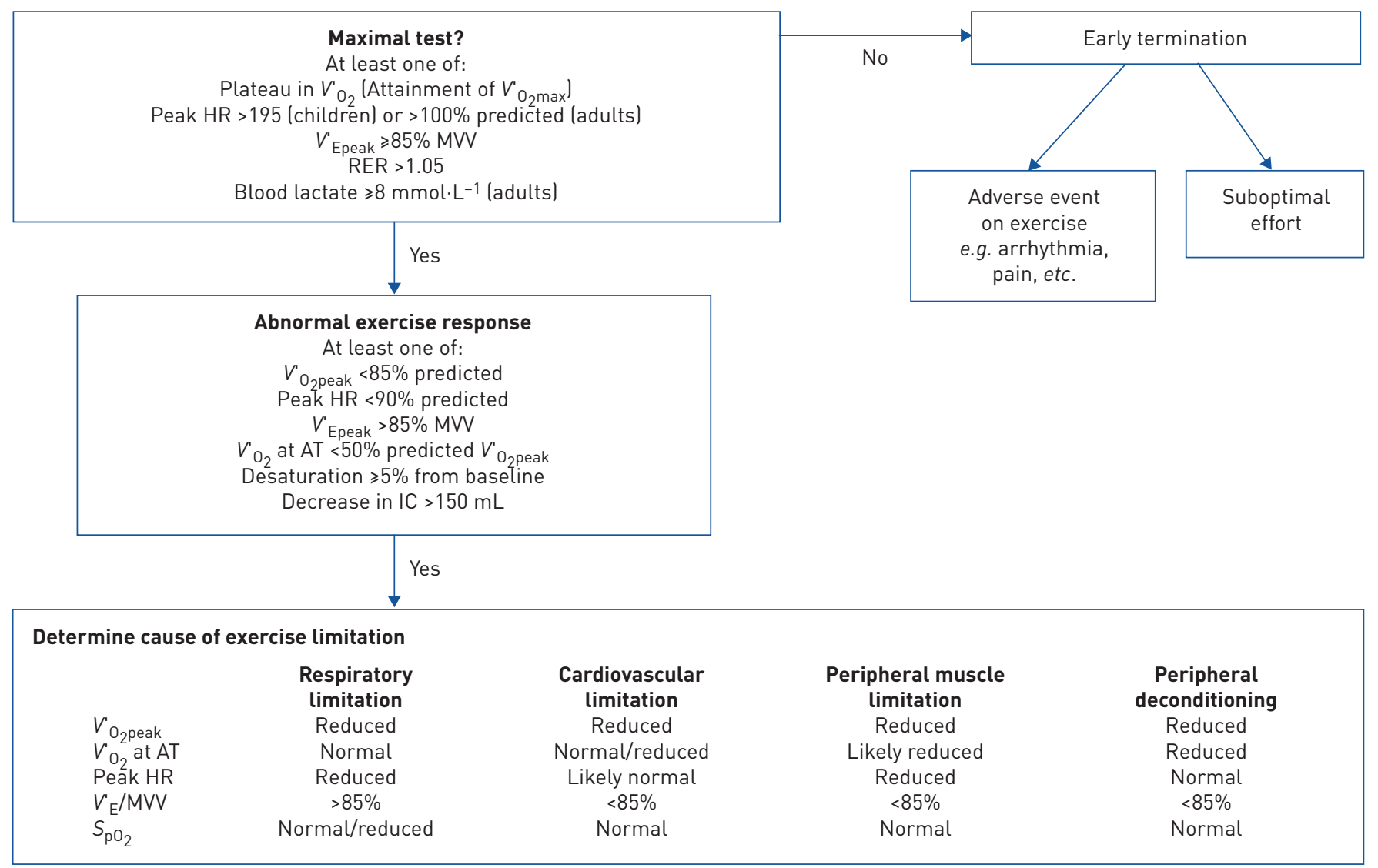

FIGURE 2 Algorithm for determining maximal effort and cause of exercise limitation. This figure describes how the Task Force members determine the maximal effort and cause of exercise limitation. It is not intended as a recommendation for clinical practice. $V^{\prime} \mathrm{O}_{2}$ : oxygen uptake; $V^{\prime}{ }_{O_{2} \text { peak }}$ : peak oxygen uptake; $V_{\text {Epeak: }}^{\prime}$ peak minute ventilation; MVV: maximum voluntary ventilation; RER: respiratory exchange ratio; HR: heart rate; AT: anaerobic threshold; IC: inspiratory capacity; $\mathrm{S}_{\mathrm{pO}_{2}}$ : arterial oxygen saturation measured by pulse oximetry.

Ventilatory limitation can be detected by comparing $V_{\text {Epeak }}^{\prime}$ to MVV. Constraints on inspiration can be detected by comparing exercise $V^{\prime}{ }_{\mathrm{T}}$ trajectory relative to exercise IC. Serial IC manoeuvres may also be valuable to detect a critical reduction in inspiratory reserve volume [55]. If IC manoeuvres could not be performed (e.g. in young children), $V_{\mathrm{T}} /$ vital capacity may give information on ventilatory limitation [11]. Moreover, evidence of a ventilatory limitation has been pointed out by the development of significant expiratory flow limitation $\left(>40 \%-50 \%\right.$ of $V_{\mathrm{T}}^{\prime}$ ) [151-154].

Determining the $V^{\prime}{ }_{\mathrm{E}} / V^{\prime} \mathrm{CO}_{2}$ slope provides relevant information especially for patients with pulmonary hypertension and heart failure $[155,156]$. Other relationships such as $V_{\mathrm{O}_{2}}^{\prime}$ /work rate and $V_{\mathrm{O}_{2}}^{\prime}$ divided by HR (=oxygen pulse) are also helpful in assessing patients with (suspected) cardiac or vascular disease [30].

\section{Clinical exercise report}

A sample summary for a report is provided in the table S14. A clinical exercise report usually consists of four components.

\section{Basic information on the patient}

The report includes name, date of birth, date of test, patient's characteristics (ECG and pulmonary function report may be added), and indication(s) for the test.

\section{Technical report}

Secondly, there is a technical report, defining protocol and work rate increment used, along with reason(s) for test termination.

\section{Response to exercise}

The report contains the reason for exercise termination, shortness of breath, leg discomfort or both. In addition, Borg scale scores may be provided at the limit of tolerance. Then, the report describes exercise responses at peak exercise and, if reliably assessable, also at AT, as well as slopes, namely the following (table S14). 
1) Aerobic/anaerobic response: $V^{\prime} \mathrm{O}_{2 \mathrm{pea}}$ rate at $\mathrm{AT}$, and $V_{\mathrm{O}_{2}}^{\prime}$ /work rate slope.

(\% predicted) and peak work rate (\% predicted), $V_{\mathrm{O}_{2}}^{\prime}$ and work

2) Cardiovascular response: $\mathrm{HR}_{\max }$ (\% predicted) and $V_{\mathrm{O}_{2}}^{\prime} / \mathrm{HR}$ (oxygen pulse) at peak exercise and at AT, along with BP and ECG responses to exercise.

3) Ventilatory response: $V^{\prime}$ Epeak and $V^{\prime}$ Epeak related to MVV. Breathing reserve and/or ventilatory limitation at maximal exercise is discussed. If available, assessment of dynamic hyperinflation by serial IC measurements is provided.

4) Gas exchange response: $S_{\mathrm{pO}_{2}}$, as well as ventilatory equivalents $\left(V^{\prime}{ }_{\mathrm{E}} / V^{\prime} \mathrm{O}_{2}, V^{\prime}{ }_{\mathrm{E}} / V^{\prime} \mathrm{CO}_{2}\right)$ and end-tidal $\mathrm{O}_{2}$ and $\mathrm{CO}_{2}$ measures at peak exercise. Report $V_{\mathrm{E}}^{\prime} / V^{\prime} \mathrm{CO}_{2}$ slope. If available, arterial blood gas analysis and $V_{\mathrm{D}} / V_{\mathrm{T}}$ (calculated from arterial carbon dioxide tension) are also added.

5) Metabolic response: RER values at rest, AT and peak exercise.

Report summary

Finally, a report summary puts the technical and exercise response information into the context of the exercising subject; e.g. What does this mean for the patient? What disease processes can be diagnosed?

\section{Data interpretation}

The data obtained from a CPET test are not interpreted in isolation. Rather, the interpretation should be an integration of CPET results with the patient's history, other clinical findings and investigations. The results of an exercise test may prompt further technical investigations (e.g. vascular assessment, assessment of respiratory or peripheral muscle function, etc.). In addition to data directly obtained from the CPET, feedback from the patient, including reason for exercise termination, can be useful in evaluating exercise limitation. Figure 2 provides a description of how Task Force members interpret CPET results.

Graphic and tabular representations of CPET variables are fundamental to appreciate the origin of system(s) limitation. Selection of the most appropriate format for data display is important for discriminating patterns of abnormality in the exercise response. Suggested forms for tabular and graphic reports of the results of incremental CPET are discussed in this and other publications $[9,11]$.

It is important to stress that the graphs chosen display the entire exercise response (rest to peak exercise and recovery) in addition to the peak values, which are also usually presented in tabular form; this enhances the interpretation. These submaximal graphic data are revealing and can often be diagnostic. The use of the appropriate corresponding normal reference values for each plot greatly enhances interpretation although the available reference data are limited [157]. Interpretation is facilitated if the tabular data are interval averaged (e.g. 10-15 s), while graphic display is often based on moving averages over four to eight breaths. For the final report, however, averages over larger intervals $(20-60 \mathrm{~s})$ were recommended by the ATS/ACCP [11]. The appropriateness or inappropriateness of the response of a variable, compared with the corresponding reference value, is the basis for determining normal or abnormal exercise responses and mechanism of exercise limitation.

\section{Information to report in exercise-related publications: protocol and outcomes}

Our rigorous systematic review identified substantial lack of information in many publications with respect to the quality of reporting on CPET equipment, testing protocols (table 2) and criteria to determine maximal effort (table 3). Based on this lack of information and the experience of the Task Force members, the following information would be useful to be reported in future studies in order to achieve standardisation of CPET in lung diseases.

\section{Equipment}

The type of ergometer (cycle ergometer or treadmill), metabolic cart, equipment used for ECG and $S_{\mathrm{pO}_{2}}$ monitoring and pulmonary gas exchange measurements (mouthpiece or facemask).

The calibration procedures if not standard (e.g. calibration gases for testing with supplemental oxygen).

\section{Exercise protocol and measurements}

The CPET protocol including duration of resting, unloaded phase (warm-up), incremental and recovery periods.

The work rate used during the unloaded phase.

The choice of work rate increments (i.e. ramp or incremental) and how the increment was determined for individual patients, if different for different patients (i.e. those with severe lung function impairment). 
The actual duration of the incremental phase (mean and standard deviation).

How the criteria were defined for reaching the limit of exercise tolerance and/or a maximal effort and how many patients actually met these criteria.

\section{Outcomes}

The following variables to describe patient characteristics (separately for different disease entities, if applicable): age, sex, height, weight, body mass index, indices of pulmonary function, $V^{\prime}{ }_{\mathrm{O}_{2 p a k}}$, peak work rate, peak $\mathrm{HR}, S_{\mathrm{pO}_{2}}$ at peak exercise, $V_{\mathrm{E}}^{\prime} / V^{\prime} \mathrm{CO}_{2}$ slope, and $V_{\mathrm{E}}^{\prime} / \mathrm{MVV}$ at peak exercise.

Additional CPET outcomes, if relevant, to describe the patient's or study outcomes.

Report the interpretation of the reason for limited exercise tolerance (i.e. cardiovascular, ventilatory, muscular and/or detraining).

\section{Results from patient survey}

There were 295 respondents from 34 countries; the majority were patients with COPD (26\%), sarcoidosis (18\%), CF (16\%) or asthma (13\%) (figure S5). The most common frequency of CPETs undertaken per patient per year was two to five $(42 \%)$, primarily on a cycle ergometer $(83 \%)$, breathing either through a facemask (52\%), mouthpiece (24\%), both on separate occasions (15\%) or other gas collection equipment (9\%) (figure S2) without routine use of supplemental $\mathrm{O}_{2}$ during testing (83\%). The total duration of CPET including preparation, resting and recovery period varied, with most being between $20-30 \mathrm{~min}$ (46\%), and $<20 \mathrm{~min}(43 \%)$; the duration of the test was "mostly acceptable" to $80 \%$ of respondents. The preferred frequency for participating in CPET in the future was once a year (45\%) or twice a year (18\%) (figure S6). When asked how much of a problem aspects of CPET were, respondents rated as a "serious problem": dryness in mouth (11\%), muscle soreness (10\%), bicycle seat uncomfortable (9\%), coughing (9\%) and mouthpiece uncomfortable (8\%). Respondents were asked about the benefits of taking part in CPET with $75 \%$ reporting improved knowledge about lung function as the greatest benefit (figure S7). Many patients perceived the CPET as a highly beneficial part of their routine screening and provided suggestions that informed this ERS Task Force. For example, suggestions made for improving the test, included: making the environment quieter, keeping patients warm, having the opportunity to sip water or to use a fan during the recovery period. They also highlighted the need for more information about the aims and benefits of the test, a detailed description of the process before taking the test as well as improved individual care during the test to include greater encouragement for their efforts and clarification for the reason for stopping the test. Following the test, patients would welcome some dedicated time to discuss the results with a healthcare professional and for any previous test results to be available for comparison.

\section{Suggestions for further work/research}

This Task Force focused on the standardisation of CPET in different pulmonary disease entities, implementing patient perspectives on CPET and describing a standard protocol for cycle and treadmill exercise testing. During the work of the Task Force, areas with limited information were identified with relevance to CPET protocol standardisation as follows.

1) Optimal selection of work rate increments during the incremental phase of the test has only been addressed in a few studies and lung diseases. Although the Task Force members provided a description of the available evidence and how to determine the increment for individual patients in this document, more studies are clearly necessary to validate the suggested approach or to provide a better method.

2) Normative data for CPET are often based on relatively small samples or specific age groups, thereby limiting their use in clinical practice. Furthermore, selecting norms from different sources hinders the comparability of CPET results between centres or within registries.

3) Comparison of different CPET procedures within the same patients (e.g. ramp versus minute-by-minute workload increments, cycle versus treadmill, impact of durations of unloaded pedalling and frequency of IC manoeuvres) on CPET outcomes.

4) High-quality test-retest reliability studies including patients with different lung diseases and a broad spectrum of disease severity to provide robust estimates on meaningful changes of CPET outcomes over time.

Future research, ideally within an ERS Task Force or a clinical collaborative scheme, may focus on the standardisation of work rate increments for different lung diseases and the collection of large CPET datasets to establish normal values for relevant CPET outcomes including, but not limited to, $V^{\prime}{ }_{\mathrm{O}_{2 p e n k}}$ in healthy subjects across a range of ages, sex and ethnicities. The focus of this work should be on cycle ergometry, since cycle ergometry is used more commonly to assess subjects with lung diseases than 
treadmill, while the data base for normative data is much smaller for cycle ergometry than for treadmill in healthy adults $[24,25]$.

\section{Summary}

This Task Force statement summarises published protocols to provide an overview of the standard practices for conducting, reporting and interpreting a CPET. Our findings provide a basis for the comparison of data from different clinical settings or published studies and identify topics that will be useful to include data in registries. Furthermore, a standardised protocol allows collection of normative data. The literature would support a test protocol with a resting phase lasting at least $3 \mathrm{~min}$, a 3-min unloaded phase (at minimal exercise intensity), and an 8- to 12-min incremental phase with work rate increased linearly at least every minute, followed by a recovery phase. The interpretation of the test requires an evaluation of whether the limits of exercise tolerance are reached. Multiple measures obtained during the test are used to address the questions triggering the test, describe abnormal responses and, if exercise limitations are detected, identifying causes for exercise limitations. The exercise report ideally includes key patient's characteristics and the indication for the test, information on test protocol and procedures, test outcomes, and a summary of the findings.

There are two areas that specifically require further research: 1) the prediction of work rate increments that result in an incremental phase of the test lasting about 10 min given the individual differences among patients in age, sex, body size, lung disease, physical activity, etc.: and 2) the collection of normative data for all CPET-derived measures that are based on large sample sizes covering the entire age range.

Author contributions: Abstract Screening: D. Berton, G. Kaltsakas, H. Hebestreit, J. De Brandt, M.A. Puhan, R.A. Rabinovich, T. Radtke, S. Crook and Z. Louvaris. Full-text screening and data extraction: A. Kampouras, C. Burtin, D. Berton, D.C.W. Braeken, D. Langer, D.S. Urquhart, F.M.E. Franssen, G. Kaltsakas, H. Hebestreit, J. De Brandt, R.A. Rabinovich, T. Radtke, S. Crook, S. Dacha, Y. Goërtz and Z. Louvaris. Management of systematic review process: T. Radtke. Statistical analysis: S. Crook. Patient survey: D. Kontopidis, J. Boyd, H. Hebestreit and I. Vogiatzis. Methodological expertise: M.A. Puhan and T. Tonia. All authors edited, critically reviewed, and approved the final version of the manuscript.

Conflict of interest: T. Radtke reports personal fees for registration and travel/accomodation for conferences from Vifor Pharma, outside of the submitted work. S. Crook has nothing to disclose. G. Kaltsakas has nothing to disclose. G. Louvaris has nothing to disclose. D. Berton has nothing to disclose. D.S. Urquhart has nothing to disclose. A. Kampouras has nothing to disclose. R.A. Rabinovich has nothing to disclose. S. Verges has nothing to disclose. D. Kontopidis is President of the Hellenic Cystic Fibrosis Association. J. Boyd reports is an employee of the European Lung Foundation. T. Tonia reports acting as ERS Methodologist. D. Langer has nothing to disclose. J. De Brandt has nothing to disclose. Y.M.J. Goërtz has nothing to disclose. C. Burton has nothing to disclose. M.A. Spruit reports grants and personal fees from Boehringer-Ingelheim and AstraZeneca, personal fees from GSK and Novartis, congress travel support from TEVA, and grants from the Netherlands Lung Foundation, outside the submitted work. D.C.W. Braeken has nothing to disclose. S. Dacha has nothing to disclose. F.M.E. Franssen reports personal fees from AstraZeneca, Boehringer Ingelheim, Chiesi, GlaxoSmithKline and TEVA, and grants and personal fees from Novartis, outside the submitted work. P. Laveneziana reports personal fees from Novartis France and Boehringer France, outside the submitted work. E. Eber has nothing to disclose. T. Troosters has nothing to disclose. J.A Neder has nothing to disclose. M.A. Puhan has nothing to disclose. R. Casaburi reports that he shares patent US 7927251 B1, relating to a linear treadmill testing protocol, issued to Los Angeles Biomedical Research Institute. I. Vogiatzis has nothing to disclose. H. Hebestreit reports grants and personal fees from Vertex Pharmaceuticals, grants from Mukoviszidose e.V. (German CF Organization), outside the submitted work; and is the Coordinator of the European Cystic Fibrosis Society Exercise Working Group who discuss exercise testing and organise respective symposia.

Support statement: Funding was received from the European Respiratory Society, grant number TF-2016-12. The research of Jana De Brandt is financially supported by FWO (grant \#11B4718N) and the research of Chris Burtin is partially sponsored by Limburg Kankerfonds. Funding information for this article has been deposited with the Crossref Funder Registry.

\section{References}

1 Hebestreit $\mathrm{H}$, Hulzebos EH, Schneiderman JE, et al. Cardiopulmonary exercise testing provides additional prognostic information in cystic fibrosis. Am J Respir Crit Care Med 2019; 199: 987-995.

2 Wensel R, Francis DP, Meyer FJ, et al. Incremental prognostic value of cardiopulmonary exercise testing and resting haemodynamics in pulmonary arterial hypertension. Int J Cardiol 2013; 167: 1193-1198.

3 Neder JA, Berton D, Rocha A, et al. Abnormal pattern of response to incremental exercise. In: Palange P, Laveneziana P, Neder JA, et al., eds. Clinical Exercise Testing (ERS Monograph). Sheffield, European Respiratory Society, 2018; pp. 34-58.

4 Neder JA, Laveneziana P, Ward SA, et al. Introduction: CPET in clinical practice. Recent advances, current challenges and future directions. In: Palange P, Laveneziana P, Neder JA, et al., eds. Clinical Exercise Testing (ERS Monograph). Sheffield, European Respiratory Society, 2018; pp. x.

5 Harvie D, Levett DZH. Exercise testing for pre-operative evaluation. In: Palange P, Laveneziana P, Neder JA, et al., eds. Clinical Exercise Testing (ERS Monograph). Sheffield, European Respiratory Society, 2018; pp. 251-279. 
Clini E, Holland AE, Pitt F, et al., eds. Textbook of Pulmonary Rehabilitation. New York, Springer International Publishing, 2018.

O'Donnell DE, Revill SM, Webb KA. Dynamic hyperinflation and exercise intolerance in chronic obstructive pulmonary disease. Am J Respir Crit Care Med 2001; 164: 770-777.

Roca J, Whipp BJ, Agusti AGN, et al. Clinical exercise testing with reference to lung diseases: indications, standardization and interpretation strategies. Eur Respir J 1997; 10: 2662-2689.

Palange P, Ward SA, Carlsen KH, et al. Recommendations on the use of exercise testing in clinical practice. Eur Respir J 2007; 29: 185-209.

Puente-Maestu L, Palange P, Casaburi R, et al. Use of exercise testing in the evaluation of interventional efficacy: an official ERS statement. Eur Respir J 2016; 47: 429-460.

American Thoracic Society/American College of Chest Physicians. Statement on cardiopulmonary exercise testing. Am J Respir Crit Care Med 2003; 167: 211-277.

Wassermann K, Hansen J, Sue D, et al. Principles of exercise testing and interpretation: including pathophysiology and clinical applications. 3rd Edn. Philadelphia, Lippincott Williams and Wilkins, 1999.

Sheehan JM, Rowland TW, Burke EJ. A comparison of four treadmill protocols for determination of maximum oxygen uptake in 10- to 12-year-old boys. Int J Sports Med 1987; 8: 31-34.

Taylor HL, Buskirk E, Henschel A. Maximal oxygen intake as an objective measure of cardio-respiratory performance. J Appl Physiol 1955; 8: 73-80.

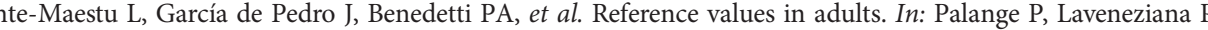
Neder JA, et al., eds. Clinical Exercise Testing (ERS Monograph). Sheffield, European Respiratory Society, 2018 pp. 88-106.

Rhodes J, Ubeda Tikkanen A, Jenkins KJ. Exercise testing and training in children with congenital heart disease. Circulation 2010; 122: 1957-1967.

Nes BM, Janszky I, Wisloff U, et al. Age-predicted maximal heart rate in healthy subjects: the HUNT fitness study. Scand J Med Sci Sports 2013; 23: 697-704.

Rowland TW. Developmental Exercise Physiology. Champaign, Human Kinetics, 1996.

Fairbarn MS, Blackie SP, McElvaney NG, et al. Prediction of heart rate and oxygen uptake during incremental and maximal exercise in healthy adults. Chest 1994; 105: 1365-1369.

Orenstein DM. Assessment of exercise pulmonary function. In: Rowland TW, ed. Pediatric Laboratory Exercise Testing Clinical Guidelines. Champaign, Human Kinetics, 1993; pp. 141-163.

Jones NL, Makrides L, Hitchcock C, et al. Normal standards for an incremental progressive cycle ergometer test. Am Rev Respir Dis 1985; 131: 700-708.

Krahenbuhl GS, Skinner JS, Kohrt W. Developmental aspects of maximal aerobic power in children. Exerc Sport Sci Rev 1985; 13: 503-538.

Armstrong N, Welsman JR. Assessment and interpretation of aerobic fitness in children and adolescents. Exerc Sport Sci Rev 1994; 22: 435-476.

Loe $\mathrm{H}$, Nes BM, Wisloff U. Predicting $\mathrm{VO}_{2}$ peak from submaximal- and peak exercise models: the Hunt 3 Fitness study, Norway. Plos One 2016; 11: e0144873.

yers J, Kaminsky LA, Lima R, et al. A Reference equation for normal standards for $\mathrm{VO}_{2}$ max: analysis from the Fitness Registry and the Importance of Exercise National Database (FRIEND Registry). Prog Cardiovasc Dis 2017; 60: 21-29.

Williams JR, Armstrong N, Kirby BJ. The $4 \mathrm{mM}$ blood lactate level as an index of exercise performance in 11-13 year old children. J Sports Sci 1990; 8: 139-147.

Armstrong N, McManus AM. Aerobic fitness. Oxford Textbook of Children's Sport and Exercise Medicine. 3rd Edn. Oxford, Oxford University Press, 2017; pp. 161-181.

Edvardsen E, Hem E, Anderssen SA. End criteria for reaching maximal oxygen uptake must be strict and adjusted to sex and age: a cross-sectional study. PLoS One 2014; 9: e85276.

Howley ET, Bassett DR, Welch HG. Criteria for maximal oxygen uptake: review and commentary. Med Sci Sports Exerc 1995; 27: 1292-1301.

Palange P, Laveneziana P, Neder J, et al., eds. Clinical Exercise Testing (ERS Monograph). Sheffield, European Respiratory Society, 2018.

Nixon PA, Orenstein DM, Kelsey SF, et al. The prognostic value of exercise testing in patients with cystic fibrosis. N Eng J Med 1992; 327: 1785-1788.

Wensel R, Opitz CF, Anker SD, et al. Assessment of survival in patients with primary pulmonary hypertension: importance of cardiopulmonary exercise testing. Circulation 2002; 106: 319-324.

Laviolette L, Laveneziana P. Exercise testing in the prognostic evaluation of patients with lung and heart diseases. In: Palange P, Laveneziana P, Neder JA, et al., eds. Clinical Exercise Testing (ERS Monograph). Sheffield, European Respiratory Society, 2018; pp. 222-234.

O'Donnell D, Elbehairy A, Berton D, et al. Exercise testing in the evaluation of pharmacotherapy in COPD. In: Palange P, Laveneziana P, Neder JA, eds. Clinical Exercise Testing (ERS Monograph). Sheffield, European Respiratory Society, 2018; pp. 235-250.

Ruf K, Hebestreit H. Exercise-induced hypoxemia and cardiac arrhythmia in cystic fibrosis. J Cyst Fibros 2009; 8: 83-90. Stuart RJ, Ellestad MH. National Survey of Exercise Stress-Testing Facilities. Chest 1980; 77: 94-97.

Fletcher GF, Ades PA, Kligfield P, et al. Exercise Standards for Testing and Training: A Scientific Statement From the American Heart Association. Circulation 2013; 128: 873-934.

Wassermann K, Hansen J, Sue D, et al. Principles of exercise testing and interpretation: including pathophysiology and clinical applications. 4th Edn. Philadelphia, Lippincott Williams and Wilkins, 2005.

Wasserman K, Whipp BJ. Exercise physiology in health and disease. Am Rev Respir Dis 1975; 112: 219-249.

Palange P, Forte S, Onorati P, et al. Ventilatory and metabolic adaptations to walking and cycling in patients with COPD. J Appl Physiol 2000; 88: 1715-1720.

Valli G, Vizza CD, Onorati P, et al. Pathophysiological adaptations to walking and cycling in primary pulmonary hypertension. Eur J Appl Physiol 2008; 102: 417-424.

Hill K, Dolmage TE, Woon L, et al. Comparing peak and submaximal cardiorespiratory responses during field walking tests with incremental cycle ergometry in COPD. Respirology 2012; 17: 278-284. 
Porszasz J, Casaburi R, Somfay A, et al. A treadmill ramp protocol using simultaneous changes in speed and grade. Med Sci Sport Exer 2003; 35: 1596-1603.

Miyamura M, Honda Y. Oxygen intake and cardiac output during treadmill and bicycle exercise. J Appl Physiol 1972; 32: 185-188.

McArdle WD, Katch FI, Pechar GS. Comparison of continuous and discontinuous treadmill and bicycle tests for $\max \mathrm{Vo}_{2}$. Med Sci Sports 1973; 5: 156-160.

Mahler DA, Gifford AH, Waterman LA, et al. Mechanism of greater oxygen desaturation during walking compared with cycling in patients with COPD. Chest 2011; 140: 351-358.

Hsia D, Casaburi R, Pradhan A, et al. Physiological responses to linear treadmill and cycle ergometer exercise in COPD. Eur Respir J 2009; 34: 605-615.

Murray JA, Waterman LA, Ward J, et al. Perceptual and physiologic responses during treadmill and cycle exercise in patients with COPD. Chest 2009; 135: 384-390.

Man WD-C, Soliman MGG, Gearing J, et al. Symptoms and quadriceps fatigability after walking and cycling in chronic obstructive pulmonary disease. Am J Respir Crit Care Med 2003; 168: 562-567.

Riddell MC, Gallen IW, Smart CE, et al. Exercise management in type 1 diabetes: a consensus statement. Lancet Diabetes Endocrinol 2017; 5: 377-390. 948-968.

Myers J, Arena R, Franklin B, et al. Recommendations for Clinical Exercise Laboratories A Scientific Statement From the American Heart Association. Circulation 2009; 119: 3144-3161.

Wilson RC, Jones PW. A comparison of the visual analogue scale and modified Borg scale for the measurement of dyspnoea during exercise. Clin Sci 1989; 76: 277-282.

Kendrick KR, Baxi SC, Smith RM. Usefulness of the modified 0-10 Borg scale in assessing the degree of dyspnea in patients with COPD and asthma. J Emerg Nurs 2000; 26: 216-222.

O'Donnel DE, Elbehairy AF, Domnik NJ, et al. Patterns of cardiopulmonary response to exercise in COPD. In: Palange P, Laveneziana P, Neder JA, eds. Clinical Exercise Testing (ERS Monograph). Sheffield, European Respiratory Society, 2018; pp. 107-127.

Guenette JA, Chin RC, Cory JM, et al. Inspiratory capacity during exercise: measurement, analysis, and interpretation. Pulm Med 2013; 2013: 956081.

Miller MR, Hankinson J, Brusasco V, et al. Standardisation of spirometry. Eur Respir J 2005; 26: 319-338.

Stickland MK, Butcher SJ, Marciniuk DD, et al. Assessing exercise limitation using cardiopulmonary exercise testing. Pulm Med 2012; 2012: 824091.

Klas JV, Dempsey JA. Voluntary versus reflex regulation of maximal exercise flow: volume loops. Am Rev Respir Dis 1989; 139: 150-156.

O'Donnell DE, Webb KA. Exertional breathlessness in patients with chronic airflow limitation. The role of lung hyperinflation. Am Rev Respir Dis 1993; 148: 1351-1357.

Whipp BJ, Davis JA, Torres F, et al. A test to determine parameters of aerobic function during exercise. J Appl Physiol Respir Environ Exerc Physiol 1981; 50: 217-221.

Myers J, Buchanan $\mathrm{N}$, Walsh $\mathrm{D}$, et al. Comparison of the ramp versus standard exercise protocols. J Am Coll Cardiol 1991; 17: 1334-1342.

Zhang YY, Johnson MC, Chow N, et al. Effect of exercise testing protocol on parameters of aerobic function. Med Sci Sports Exerc 1991; 23: 625-630.

Benzo RP, Paramesh S, Patel SA, et al. Optimal protocol selection for cardiopulmonary exercise testing in severe COPD. Chest 2007; 132: 1500-1505.

Hartman JE, Slebos DJ, Boezen HM, et al. Selecting the increment size for a maximal incremental cycle test in patients with COPD. Respirology 2015; 20: 352-355.

Hulzebos HJ, Werkman MS, van Brussel M, et al. Towards an individualized protocol for workload increments in cardiopulmonary exercise testing in children and adolescents with cystic fibrosis. J Cyst Fibros 2012; 11: $550-554$.

Ochmann U, Kotschy-Lang N, Raab W, et al. Is an individual prediction of maximal work rate by 6-minute walk distance and further measurements reliable in male patients with different lung diseases? Respiration 2013; 86: 384-392.

Balady GJ, Arena R, Sietsema K, et al. Clinician's Guide to Cardiopulmonary Exercise Testing in Adults A Scientific Statement From the American Heart Association. Circulation 2010; 122: 191-225.

Bruce RA, Kusumi F, Hosmer D. Maximal oxygen intake and nomographic assessment of functional aerobic impairment in cardiovascular disease. Am Heart J 1973; 85: 546-562.

Nagle FJ, Balke B, Naughton JP. Gradational step tests for assessing work capacity. J Appl Physiol 1965; 20: $745-748$.

Naughton J, Sevelius G, Balke B. Physiological responses of normal and pathologic subjects to a modified work capacity test. J Sports Med Phys Fitness 1963; 31: 201-207.

Holm SM, Rodgers W, Haennel RG, et al. Effect of modality on cardiopulmonary exercise testing in male and female COPD patients. Resp Physiol Neurobiol 2014; 192: 30-38.

Crisafulli E, Vigna M, Ielpo A, et al. Heart rate recovery is associated with ventilatory constraints and excess ventilation during exercise in patients with chronic obstructive pulmonary disease. Eur J Prev Cardiol 2018; 25: 1667-1674

Lacasse M, Maltais F, Poirier P, et al. Post-exercise heart rate recovery and mortality in chronic obstructive pulmonary disease. Respir Med 2005; 99: 877-886.

Ramos RP, Arakaki JSO, Barbosa P, et al. Heart rate recovery in pulmonary arterial hypertension: relationship with exercise capacity and prognosis. Am Heart J 2012; 163: 580-588.

Borg G. Perceived exertion as an indicator of somatic stress. Scand J Rehabil Med 1970; 2: 92-98.

Borg GA. Psychophysical bases of perceived exertion. Med Sci Sports Exerc 1982; 14: 377-381.

Gift AG. Visual analogue scales: measurement of subjective phenomena. Nurs Res 1989; 38: 286-288.

Troosters T, Verstraete A, Ramon K, et al. Physical performance of patients with numerous psychosomatic complaints suggestive of hyperventilation. Eur Respir J 1999; 14: 1314-1319. 
Kligfield P, Gettes LS, Bailey JJ, et al. Recommendations for the standardization and interpretation of the electrocardiogram: part I: The electrocardiogram and its technology: a scientific statement from the American Heart Association Electrocardiography and Arrhythmias Committee, Council on Clinical Cardiology; the American College of Cardiology Foundation; and the Heart Rhythm Society: endorsed by the International Society for Computerized Electrocardiology. Circulation 2007; 115: 1306-1324.

111 Rautaharju PM, Prineas RJ, Crow RS, et al. The effect of modified limb electrode positions on electrocardiographic wave amplitudes. J Electrocardiol 1980; 13: 109-113.

112 Kleiner JP, Nelson WP, Boland MJ. The 12-lead electrocardiogram in exercise testing. A misleading baseline? Arch Intern Med 1978; 138: 1572-1573.

113 Perloff D, Grim C, Flack J, et al. Human blood pressure determination by sphygmomanometry. Circulation 1993; 88: 2460-2470.

114 Yamamoto Y, Takei Y, Mokushi K, et al. Breath-by-breath measurement of alveolar gas-exchange with a slow-response gas analyzer. Med Biol Eng Comput 1987; 25: 141-146.

115 Yeh MP, Adams TD, Gardner RM, et al. Turbine flowmeter $v s$ fleisch pneumotachometer - a comparative-study for exercise testing. J Appl Physiol 1987; 63: 1289-1295.

116 Yoshiya I, Shimada Y, Tanaka K. Evaluation of a hot-wire respiratory flowmeter for clinical applicability. J Appl Physiol Respir Environ Exerc Physiol 1979; 47: 1131-1135. 


$$
\text { Com }
$$
Co Committee and the HICPAC/SHEA/APIC/IDSA Hand Hygiene Task Force. Society for Healthcare Epidemiology of America/Association for Professionals in Infection Control/Infectious Diseases Society of America. MMWR Recomm Rep 2002; 51: 1-45.

134 World Health Organization. Practical Guidelines for Infection Control in Health Care Facilities. SEARO Regional Publication No. 41. Geneva, WHO, 2018.

135 Rutala WA, Weber JA, Healthcare Infection Control Practices Advisory Board (HICPAC). Guideline for Disinfection and Sterilization in Healthcare Facilities, 2008. Center for Disease Control and Prevention, 2018. www. cdc.gov/infectioncontrol/guidelines/disinfection Date last updated: May 2019. Date last accessed: 31 January 2019.

136 Kendrick AH, Johns DP, Leeming JP. Infection control of lung function equipment: a practical approach. Respir Med 2003; 97: 1163-1179.

137 Hebestreit H, Arets HG, Aurora P, et al. Statement on Exercise Testing in Cystic Fibrosis. Respiration 2015; 90: 332-351.

138 Campbell SC. A comparison of the maximum voluntary ventilation with the forced expiratory volume in one second: an assessment of subject cooperation. J Occup Med 1982; 24: 531-533.

139 Dillard TA, Hnatiuk OW, McCumber TR. Maximum voluntary ventilation. Spirometric determinants in chronic obstructive pulmonary disease patients and normal subjects. Am Rev Respir Dis 1993; 147: 870-875.

140 Stein R, Selvadurai H, Coates A, et al. Determination of maximal voluntary ventilation in children with cystic fibrosis. Pediatr Pulmonol 2003; 35: 467-471.

141 Kor AC, Ong KC, Earnest A, et al. Prediction of the maximal voluntary ventilation in healthy adult Chinese subjects. Respirology 2004; 9: 76-80.

142 Gift AG. Validation of a vertical visual analogue scale as a measure of clinical dyspnea. Rehabil Nurs 1989; 14: 323-325.

143 Grant S, Aitchison T, Henderson E, et al. A comparison of the reproducibility and the sensitivity to change of visual analogue scales, Borg scales, and Likert scales in normal subjects during submaximal exercise. Chest 1999; 116: $1208-1217$.

144 Grant S, McMurray J, Aitchison T, et al. The reproducibility of symptoms during a submaximal exercise test in chronic heart failure. Br J Clin Pharmacol 1998; 45: 287-290.

145 Hamilton AL, Killian KJ, Summers E, et al. Symptom intensity and subjective limitation to exercise in patients with cardiorespiratory disorders. Chest 1996; 110: 1255-1263.

146 Agostoni P, Cattadori G. Pattern of cardiopulmonary response to exercise in cardiac disease. In: Palange P, Laveneziana P, Neder JA, et al., eds. Clinical Exercise Testing (ERS Monograph). Sheffield, European Respiratory Society, 2018; pp. 146-159.

147 Wasserman K, Whipp BJ, Koyl SN, et al. Anaerobic threshold and respiratory gas exchange during exercise. J Appl Physiol 1973; 35: 236-243.

148 Beaver WL, Wasserman K, Whipp BJ. Bicarbonate buffering of lactic acid generated during exercise. J Appl Physiol 1986; 60: 472-478.

149 Patessio A, Casaburi R, Carone M, et al. Comparison of gas exchange, lactate, and lactic acidosis thresholds in patients with chronic obstructive pulmonary disease. Am Rev Respir Dis 1993; 148: 622-626.

150 Roman MA, Casaburi JD, Porszasz J, et al. Noninvasive assessment of normality of VD/VT in clinical cardiopulmonary exercise testing utilizing incremental cycle ergometry. Eur J Appl Physiol 2013; 113: 33-40. 
151 Johnson BD, Reddan WG, Pegelow DF, et al. Flow limitation and regulation of functional residual capacity during exercise in a physically active aging population. Am Rev Respir Dis 1991; 143: 960-967.

152 Johnson BD, Saupe KW, Dempsey JA. Mechanical constraints on exercise hyperpnea in endurance athletes. J Appl Physiol 1992; 73: 874-886.

153 Johnson BD, Scanlon PD, Beck KC. Regulation of ventilatory capacity during exercise in asthmatics. J Appl Physiol 1995; 79: 892-901.

154 Johnson BD, Weisman IM, Zeballos RJ, et al. Emerging concepts in the evaluation of ventilatory limitation during exercise: the exercise tidal flow-volume loop. Chest 1999; 116: 488-503.

155 Farina S, Correale M, Bruno N, et al. The role of cardiopulmonary exercise tests in pulmonary arterial hypertension. Eur Respir Rev 2018; 27: 170134.

156 Corra U, Agostoni PG, Anker SD, et al. Role of cardiopulmonary exercise testing in clinical stratification in heart failure. A position paper from the Committee on Exercise Physiology and Training of the Heart Failure Association of the European Society of Cardiology. Eur J Heart Fail 2018; 20: 3-15. 